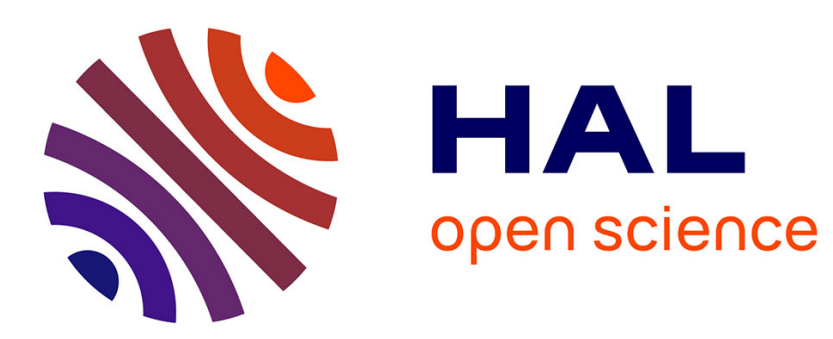

\title{
Solid State Chemistry: Computational Chemical Analysis for Materials Science
}

Estelina Lora da Silva, Sandra Galmarini, Lionel Maurizi, Mario Jorge Cesar dos Santos, Tao Yang, David J Cooke, Marco Molinari

\section{- To cite this version:}

Estelina Lora da Silva, Sandra Galmarini, Lionel Maurizi, Mario Jorge Cesar dos Santos, Tao Yang, et al.. Solid State Chemistry: Computational Chemical Analysis for Materials Science. Computational Techniques for Analytical Chemistry and Bioanalysis, pp.287-334, 2020, 10.1039/978178801588200287 . hal-03276186

\section{HAL Id: hal-03276186 \\ https://hal.science/hal-03276186}

Submitted on 1 Jul 2021

HAL is a multi-disciplinary open access archive for the deposit and dissemination of scientific research documents, whether they are published or not. The documents may come from teaching and research institutions in France or abroad, or from public or private research centers.
L'archive ouverte pluridisciplinaire HAL, est destinée au dépôt et à la diffusion de documents scientifiques de niveau recherche, publiés ou non, émanant des établissements d'enseignement et de recherche français ou étrangers, des laboratoires publics ou privés. 
Book Title: Computational Techniques for Analytical Chemistry and Bioanalysis. In Part B. Applications

Chapter Title: Solid State Chemistry: Computational Chemical Analysis for Materials Science

\title{
Authors:
}

Estelina Lora da Silva, Instituto de Diseño para la Fabricación y Producción Automatizada, and MALTA Consolider Team, Universitat Politècnica de València, Camí de Vera s/n, 46022 València, Spain. Tel: +34-645825531. esdasil@idf.upv.es. ORCID 0000-0002-7093-3266

Sandra Galmarini, Empa Materials Science and Technology: Dubendorf, ZH, Switzerland. Tel: +41 58765 4066, Sandra.Galmarini@empa.ch. ORCID 0000-0003-2183-3100

Lionel Maurizi, Laboratoire Interdisciplinaire Carnot de Bourgogne, UMR 6303 CNRS - Université Bourgogne Franche-Comté, BP 47870, F-21078 Dijon cedex, France. Tel: +333 803961 71, lionelmaurizi@gmail.com, ORCID 0000-0002-6346-7623

Mario Jorge Cesar dos Santos, Departamento de Fisica, Faculdade de Ciencias e Tecnologias, Universidade de Coimbra, R. Larga, 3004-516 Coimbra, Portugal. Tel: +00351 936911449. mario.cesar.santos@ua.pt. ORCID 0000-0002-3114-7473

Tao Yang, TEMA-NRG, Department of Mechanical Engineering, University of Aveiro, 3810-193 Aveiro, Portugal. Tel: +351 967085271, yangtao@ua.pt, ORCID 0000-0002-4053-124X

David J. Cooke, Department of Chemical Sciences, School of Applied Sciences, University of Huddersfield, Queensgate, Huddersfield HD1 3DH, United Kingdom. Tel: +44 1484 472703, d.j.cooke@hud.ac.uk, ORCID 0000-0001-5996-7900

Marco Molinari, Department of Chemical Sciences, School of Applied Sciences, University of Huddersfield, Queensgate, Huddersfield HD1 3DH, United Kingdom. Tel: +44 1484472973 , m.molinari@hud.ac.uk, ORCID 0000-0001-7144-6075

\begin{abstract}
We present an overview of computational analytical methodologies and protocols applied to materials analysis, and their surfaces and interfaces with the surrounding environments. As we discuss the current advances and limitations of in-silico measurements applied to materials science, we highlight their complementary achievements and their innovative predictions in the view of their experimental counterparts. We focus on elemental, structural and chemical analyses of complex advanced materials, either homogeneous or heterogeneous targets.
\end{abstract}

Table of Content this will be updated automatically in my version

1. Computational Spectroscopy - Interaction between matter and electromagnetic radiation. This section will cover the application of these techniques to bulk, surfaces, interfaces and nanoparticles.

a. Emission spectroscopy. 
b. Adsorption spectroscopy: (1) X-ray Absorption Spectroscopy (XAS - including Extended $X$-Ray Absorption Fine Structure (EXAFS) and X-ray Absorption Near Edge Structure (XANES)), (2) UV/Vis, (3) IR

c. Inelastic scattering: (1) Raman Spectroscopy, (2) Electron Energy Loss Spectroscopy (EELS)

d. Elastic scattering: X-ray diffraction

e. Coherent or resonance spectroscopy: (1) Nuclear Magnetic Resonance (NMR) or Magnetic Resonance Spectroscopy (MRS): observe local magnetic fields around atomic nuclei, (2) Electron Spin Resonance (ESR) or Electron Paramagnetic Resonance (EPR): study materials with unpaired electrons, (3) Muonium spectroscopy ( $\mu S R)$ : local environments, (4) Positron Annihilation Spectroscopy: study voids, vacancies, pores - by employing two-component DFT (available in ABINIT and a VASP interface MIKKA Doppler)

2. Computational Analysis of Biocompatible Materials. This section will focus on the concept of "interface" with focus on the interactions between the surfaces of materials and the biological environment. We will explain the scientific questions and focus on the computational analytical techniques that can measures these properties at the interfaces.

a. Surface properties. (1) Segregation of defects, electrical double layer. (2)Restructuration/Hydroxylation etc. (3) Growth/dissolution (surface roughness)

b. Surface adsorption and surface functionalization (molecules) (1) Surface functionalization by grafting or voluntary adsorption of molecules. (2) Adsorption of biomolecules in biological media. (3) Nanoparticles: Size, Aggregation/Agglomeration

c. Influence of size at the bio-interface (1) Agglomeration / Colloidal forces - Brownian motion, vdW, electrostatic, magnetic. (2) Micelle formation 


\section{Introduction}

The development and production of materials with various physico-chemical properties for specific applications, especially for medical and biological purposes, has seen a lightning growing these last decades. Among many promising functionalities, materials can be used to improve, cure or prevent human diseases or weaknesses. For instance, metallic materials are providing functionalities in antimicrobial and therapeutic compounds such as coatings on medical devices or implants. Synthesized materials can also repair or replace defected body parts. The nanotechnologies, meanwhile, are offering promising applications in diagnoses or specific targeting. However and despite all their potential biomedical applications and their development focusing on biocompatible materials are still in their infancy.

An interplay between experimental and computational techniques is necessary to achieve a full understanding of biochemical analysis. Whereas this chapter aims to cover the Solid State Chemistry within the biochemical remit, highlighting the advantages of such computational analysis, its exploitation within the biological community is still quite limited. We therefore will cover those techniques that are currently at the forefront of the materials science, but still have not seen their full application in biochemistry.

This need to be expanded - at least another paragraph spelling out what we cover and why (in general terms). Marco to do it.

2 Computational Spectroscopy. The Interaction between matter and electromagnetic radiation.

This section will focus on some of the most relevant computational methodologies that can be applied to probe the spectroscopic properties of systems for medical and biological applications. However before discussing such techniques, we have highlighted some relevant systems and their applications in biomedicine. Computational methodologies to study such systems will be discussed within the experimental spectroscopy data in each relevant subsection.

\subsection{Biomedical Applications and Relevance of Spectroscopy}

\subsubsection{Zinc Oxide Nanoparticles}

Transition metal oxides, with their incomplete valence electron of the $d$-shells, are responsible for forming intermediate chemical bonding.[1] This variety of bonding configurations allows transition metal oxides to explore different crystal structures under specific temperature and pressure conditions; they may exhibit several phases including spin, charge, orbital states, which enhance further the diversity of applications.[2]

The plethora of applications can be evidenced with a simple example of nanoparticles (NPs) of $\mathrm{ZnO}$, which have been shown to exhibit excellent properties for orthopaedic and dental implants,[3] drug delivery, anti-cancer, anti-diabetic, antibacterial, antifungal and biological sensing applications.[4] Through electrohydrodynamic atomisation of ZnO NPs as a coating material, bacterial adhesion can 
be inhibited and osteoblast growth promoted.[3] ZnO NPs have also established their capability in biological imaging, due to their high nonlinear optical coefficients; in fact, have been used for in vitro imaging of living cells.[5] Moreover, and since ZnO NPs possess large band-gaps (3.37eV) with high exciton binding energies $(60 \mathrm{meV})$, promotion of high catalytic efficiency is enabled, which is a desirable character for biosensing.[4]

Development of novel nano-methodologies to promote alternative approaches for antimicrobial applications has led to the development of multifunctional agents with tailored physiochemical properties (shape, increased surface area and size, combined with their toxic nature towards prokaryotic organisms).[6] Alternatives to $\mathrm{ZnO}$ have been developed as dielectric films or laminar structures fabricated via atomic layer deposited of metal oxides such as $\mathrm{HfO}_{2}, \mathrm{TiO}_{2}, \mathrm{ZrO}_{2}, \mathrm{Al}_{2} \mathrm{O}_{3}$. They have all shown surprisingly efficient antibacterial activity, comparable to the properties of $\mathrm{ZnO}$.[2] However, the advantage of low temperature layer deposition (below $100^{\circ} \mathrm{C}$ ) has opened the possibility of coating several types of perishable substrates, including soft tissue paper, cloth, and fabric for surgical masks and wound dressing, surgical instruments, door handles, as well as synthetic and organic materials for medicine, veterinary, health care and food industry supply.

\subsubsection{Graphene-like Materials}

Carbon nanotechnology is currently at the forefront of biomedical application although generally utilized in conjunction with existing biocompatible systems. Graphene-like systems (i.e. two or more layers of graphene) have proved efficient in terms of biomedical applications. Graphene and its derivatives have excellent mechanical and lubricant properties (decreasing friction),[7] enhanced capacity for antibacterial properties and biocompatibility.[8] Some applications include medical bone implants, mainly when coated onto ceramic biosurfaces, such as Ti surfaces embedded with $\mathrm{SiC},[7]$ or Hydroxyapatite (HaP).[9] HaP on its own is a biocompatible bioceramic for hard tissue repair and regeneration, due to its similarities to natural apatite. However, HaP has some drawbacks, including fracture toughness, poor tensile strength and weak wear resistance, hence reinforcement films such as graphene (or derivatives), can be implemented in order to produce biocompatible composites with increased resistance and strength.[9] $\mathrm{HaP}$ also demonstrates favourable affinity for bacterial adhesion[10] and therefore graphene-like coatings can provide the biosurface with antibacterial or bacteriostatic protection.[9]

Graphene has shown promising inexpensive, reliable, high-throughput sequencing with application in nanopore-based DNA sequencing.[11] [12] The monoatomic thickness of $0.35 \mathrm{~nm}$ is similar to the DNA base spacing and graphene nanopores can be fabricated with a diameter of only $1.0 \mathrm{~nm}$, which is about the size of a DNA sequence.

Further biological applications for graphene are in neuroscience. Uncoated graphene can be used as neuro-interface electrode without altering or damaging properties such as signal strength or formation of scar tissue. Graphene electrodes in the body are significantly more stable than electrodes of tungsten or silicon because of properties such as flexibility, biocompatibility and conductivity.[13] 
When biosurfaces are coated with graphene, it is possible to generate a small electrical impulse through the piezoelectric effect, thus stimulating cellular and bone regeneration. In graphene bilayers, it is the existence of two stacking arrangements of individual layers that allows for the possibility of this electrical impulse generation.[14] Through the 'sliding' between these layers, transitioning from the AA-phase to the AB-phase (e.g. via the movement of a joint) electrical dipoles are generated. This effect can occur due to tension and/or pressure applied between layers during the phase transition where piezoelectric effects become dominant.[15] Furthermore, the application of strain between two graphene layers enables charge transfer between the layers,[16] enhancing the intensity of dipolar interaction between the graphene and the biosurface. The variety of distortion in graphene bilayers can involve also small angular distortions,[17] which generates systems known as twisted bilayer graphene. Similar to the sliding of graphene layers, the twisting enables the emergence of piezoelectric effects as the rotation of adjacent layers occurs in order to minimize the tension between the layers themselves.

The underlying idea of generating the piezoelectric effect generated by sliding or rotation of the graphene layers, can also be applied when graphene is adsorbed on biosurfaces, e.g. graphene deposited on a $\mathrm{Si} / \mathrm{Si} \mathrm{SiO}_{2}$ surface.[18] At the nanoscale, the electric dipole moments (piezoelectric effect) are generated due to the strain induced by the surface corrugations and the difference between the lattice topology of the two materials surfaces.[18] [19] Moreover, the addition of graphene to a host biomaterial has the potential to increase the conductivity if uniformly incorporated, even at low vol\% due to its high intrinsic conductivity; unique properties of graphene can be transferred to the host surface for biomechanical applications.[20]

\subsubsection{Bulk vs Nanoparticle}

The importance of the properties that can be exploited for biomedical applications imposes the gathering of a complete characterisation of the nanodevice systems. This should be applied to both bulk and nanostructures, as they possess such different properties from those of bulk materials (e.g. the confinement excitonic effects strongly affects the optical properties), by probing the corresponding structural, electronic, optical, magnetic and dynamical properties. This characterisation includes the study of the most common and abundant impurities and chemical defects found in these systems, i.e. hydrogen impurities and native defects (i.e. vacancies), since these alter the chemical properties of interest. An interesting example is observed for Cerium Oxide (ceria) which can exist in two forms in the bulk state: $\mathrm{CeO}_{2}$ and $\mathrm{Ce}_{2} \mathrm{O}_{3}$, due to the transformation of $\mathrm{Ce}^{4+}$ to $\mathrm{Ce}^{3+}$ being intermediated by oxygen vacancies. However in the nanostructure form, a mixture of $3+$ and $4+$ states of Ce exist on the surface of the nanostructure. When the diameter of the nanostructure decreases, the number of $3+$ sites increase, as well as the $O$ vacancies, enabling the NPs to absorb and release $O$ due to the redox-cycling between the two charged states. This property ensures that ceria NPs can be used in pharmacological and biological applications.[21] A more complete description of these nanosystems and computational techniques to study such systems will be detailed on Section 3.3. 


\subsection{Absorption Spectroscopy for the Evaluation of Opto-Electronical Properties}

Adsorption computational spectroscopy can provide insights into the mechanisms underlying the excitation processes at the atomistic level, and providing fundamental understanding to direct the design and optimisation of materials for biomedical applications. This is generally done using abinitio calculations within the framework of density functional theory (DFT).[2] [22] Such level of theory can be applied to both quantities related to the electronic ground-state (time-independent DFT) and to excited states (time-dependant DFT).

Computational spectroscopy allows for the prediction of accurate values of fundamental optoelectronical properties (gap, absorption spectra, excitons) and simulate structural and dynamical properties of materials with interest for biomedical application (nanostructured systems, large unitcells, defects, doping, interfaces). For examples, vibrational spectroscopy such as IR absorption and Raman scattering has served as a fundamental tool for determining the weak interactions between guanine (DNA base adsorption) on $\mathrm{ZnO}$ model clusters $\left(\mathrm{g}-\mathrm{Zn}_{\mathrm{n}} \mathrm{O}_{\mathrm{n}}\right.$ ) and comparison performed between DFT data. Adsorption of guanine with a $\mathrm{ZnO}$ cluster has been theoretically probed in terms of geometry, binding energies, electronic and spectral properties, HOMO-LUMO charge distribution and Mulliken charges. Guanine favour physisorption with weak $\mathrm{Zn}-\mathrm{N}$ bonds tend to form on the active $\mathrm{Zn}^{2+}$ site.[23]

\subsubsection{A Brief Overview of the Theory}

Based on first-principles calculations, a system of interacting electrons and nuclei in the presence of time-dependent external fields can be calculated by employing several different methods, depending on their accuracy. The most widely used are Density based methods, such as the Time-Dependent extension of DFT to access neutral excitations,[24] Green's function based methods, the many-body perturbation theory (GW[25] and BSE[26]).

Time-dependent (TD-)DFT allows the computation of excited state properties, since the respective Kohn-Sham scheme obeys a one-particle Schrödinger time-dependent equation. Within this approach, the absorption spectra is obtained through a linear change of the density produced by a small change in the external potential, which is the linear response theory. However, and similarly to the ground-stated DFT, the time-dependent exchange-correlation (xc) and its first derivative, the exchange-correlation kernel $\left(f_{x c}\right)$, encompass all non-trivial many-body effects, which have to be obtained by different approximations (28). The most common approximation to the $\mathrm{f}_{\mathrm{xc}}$ kernel is the adiabatic local-density approximation (ALDA), where it is assumed that at time $t$, the kernel is equal to the ground-state LDA potential, obtained with the density $n(r, t)$. ALDA fails to describe the absorption spectra of extended systems, especially for wide band-gap semiconductors. ALDA, does not satisfy the divergence of $1 / \mathbf{k}^{2}$ for small wave-vectors, and instead converges to a constant value. Some successful kernels have currently been implemented (long-range corrected kernel, bootstrap 
kernel), which account for the correct divergence behaviour, however, these fail to capture excitonic effects.[27]

Many-body perturbation theory (MBPT) is a method based on the Green's function method, and provides a more accurate approach to obtaining excited state properties.[28] The one-particle Green's function, $G\left(\mathbf{r}, t ; \mathbf{r}^{\prime}, \mathrm{t}^{\prime}\right)$, can be thought of being the probability amplitude for the propagation of an additional particle from $\left(\mathbf{r}^{\prime}, \mathrm{t}^{\prime}\right)$ to $(\mathbf{r}, \mathrm{t})$ in a many-body interacting system, thus taking into account an ensemble consisting of a particle interacting with surrounding opposite charges - quasiparticle (QP).[28] Quasiparticle energies are calculated within the GW approximation, in which the expansion of the self-energy, $\Sigma$, in terms of $G$ and screened Coulomb interaction $W, \Sigma=G W$, is truncated after the first term. Since the GW approximation considers the addition and/or removal of an electron (one-particle $\mathrm{G}$ ) the one-particle excitation spectra can be computed. The experimental observable is related to direct or inverse photoemission,[28] which provides information regarding the band-gap widths and characters of the valence and conduction band states.

Within MBPT, a method to compute the optical response is through the Bethe-Salpeter equation (BSE). Since the BSE is a process involving two-particle excitations (two-particle G),[26] [28] this method accounts for electron-hole interactions (excitonic effects), therefore prevailing over the noninteracting TD-DFT methodology. Since optical absorption experiment creates this electron-hole interaction (the exciton), good agreement between theory and experiment can be achieved by taking into account the exciton. This effect is mainly important when considering small-gap semiconductors and metals, since these materials screen excitons.[26] However, to obtain such theoretical accuracy, one has to consider that the two-particle nature of the BSE makes the calculations computationally expensive, since a four-point equation (due to the propagation of two particles, starting and end points) has to be solved.[26] The GW method, combined with the solution of the Bethe-Salpeter equation (BSE), is currently the most reliable approach to calculate optical response functions.

\subsubsection{Optical Properties of Materials and Dependence on their Dimensionality}

The optical properties of macroscopic (bulk) materials emerge from the induced polarisation when the material is subject to an external time-dependent electromagnetic field and macroscopic susceptibilities. By expanding the induced polarisation, the linear term becomes the macroscopic spontaneous polarisation and the non-linear effects come about from the second, third and higher order terms of the expansion.[29] For extended systems the equations of motion and the coupling of the electrons with the external electric field are derived from the Berry-phase formulation of the dynamical polarisation.[30] It is then possible to calculate the second-and the third-harmonic generation of systems under interest. This formulation is developed upon the Green's function method. However within TD-DFT, it is also possible to evaluate the second harmonic generation (SHG) in semiconductors which also takes into account the crystal local-field and excitonic effects.[31] However, the later method is limited to the treatment of the electron correlation of systems with weakly bound excitons. 
TD-DFT has been successfully applied to $\mathrm{ZnO}$ clusters to probe respective optical properties (and validated by employing and MBPT).[32] The absorption spectra has shown an enhancement in the intensity with a blueshift of the spectral lines occurring with an increase in the cluster size. The observed optical gap was closely dependent on the size, geometrical shape, symmetry and dimensionality of the structures. From inspection of molecular orbitals and eigenvalues, it was shown that the electronic transitions occur between the non-bonding $\mathrm{O} p$-states and unoccupied $\mathrm{Zn} s p$ hybrid orbitals.

It is also possible to express the optical properties of finite systems, such as atoms, molecules, or clusters, within the dipole approximation (as the field-induced dipole polarisation[29]), where the microscopic polarisability is defined by the linear term and the first and second hyperpolarisabilities are the non-linear contributions to the optical properties.[29] [33] At the microscopic level the quadratic dependence on the field means that the magnitude of the quadratic hyperpolarisability tensor must be enhanced in order to produce improved SHG characteristics. This effect is produced when two photons create a single photon of twice the energy of the original two photons. [5] Materials displaying non-linear optical (NLO) properties are important not only for optoelectronics applications but also for sensing and bioimaging. ZnO NPs have shown efficient NLO properties which enables efficient applications for in vitro imaging of living cells.[5] Moreover, it has been shown that ZnO NPs display pronounced nonlinear doubling effect (SHG).[5]

Graphene provides a similar functionality for biosensing applications and has been successfully exploited for protein binding sensor platforms.[34] In graphene, a further advantage is the tenability of the nonlinear third-harmonic generation (frequency tripling) using an electric gate voltage.[35] This technique has particular promise in biomedicine as this provides an alternative avenue for real-time imaging of histopathological quality; examples include the characterisation of tumor tissue via realtime optical biopsies.[36] The conventional technique that has been applied successfully to detect brain tumor tissue is stimulated Raman microscopy. However, such a technique is based on subtle differences in the vibrational spectra of the tumor tissue and healthy tissue, thus requiring extensive comparison of experimental spectra against libraries of reference spectra.[36]

\subsection{Inelastic scattering: Lattice Dynamics and Raman Spectroscopy}

Carbon nanotechnology have shown great promise in biomedical applications. All from nanotubes to fullerenes passing through graphene have been shown to be promising nanozymes. Here we show the computational application of Raman spectroscopy applied to graphene, which conceptually can be extended to any biochemical system that contains graphene. The application of Raman spectroscopy allows for the identification of monolayer or multi-layered graphenes in the Bernal (AB) configuration.[37] As graphene samples are produced in bulk both via exfoliation or epitaxial methods, with both techniques giving a variety of graphene rotational disorder,[37] each of these permutations will impact on the biochemical property of the systems. Hence, the characterization of 
such configurations becomes of paramount importance to control selectively graphene behaviour in biomedical systems.

Within the Harmonic Approximation, lattice dynamics enables the computation of the vibrational modes (phonons) at the zone-centre, which can be directly compared with experimentally derived Raman spectroscopy. Bilayer graphene consists of two coupled monolayers of carbon atoms, each with a honeycomb crystal structure. Although, we focus on biomedical applications, the very basic of this technique resides in the physics at the atomistic scale that describes the vibrational motion of the structure. In order to satisfy the translational and symmetry properties of the Bravais lattice, the honeycomb graphene lattice can be seen as two sublattices, mathematically labelled as inequivalent $A$ and $B$ lattices, each containing two atoms. Since in bilayer graphene there are two lattices ( $A$ and $B)$, then there are 4 inequivalent carbon atoms that need a description within the unit cell, this leads to three acoustic $(\mathrm{A})$ and $9(3 \mathrm{~N}-3)$ optical $(\mathrm{O})$ lattice vibrations or phonon branches.[14]

Comparison between LDA calculated graphene phonon dispersions with the in-plane phonon dispersion of graphite obtained from inelastic X-ray scattering displays reasonable agreement (Figure 1), although a small shift of the higher-frequency transverse optic (TO) and longitudinal optic (LO) modes is observed. LDA calculations often overestimate the energies of higher-frequency phonons, but despite this difference the characteristic features of the phonon dispersion are well reproduced. At low q-vectors, the in-plane transverse acoustic (TA) and longitudinal acoustic (LA) modes show linear dispersions. While the doubly-degenerate LA mode has zero frequency at the $\Gamma$ point, the TA mode, also known as the shear-mode (Figure 2), has a non-zero frequency at the zonecentre ( $v=0.82 \mathrm{THz}$ ).[38] The ZA mode is the flexural acoustic mode (Figure 2), which corresponds to out-of-plane, in-phase atomic displacements. In contrast to the TA and LA modes, the ZA branch shows a parabolic dispersion close to the $\Gamma$-point, indicating low group velocity and being a characteristic feature of layered structures.[38] The existence of a flexural mode is also a signature of $2 \mathrm{D}$ systems, and in particular it is a mode which is typically found in graphene-like systems. Since the long-wave flexural mode has the lowest frequency, it is the easiest to excite.[39] The flexural mode is relevant for the understanding the intrinsic properties of graphene (i.e. electrical resistivity and thermal expansion coefficient). For example, for monolayer graphene, the high thermal conductivity is due to the vibrational morphology of the flexural mode.[39] When graphene-like systems are adsorbed on biosurfaces, this flexural mode tends to hybridize with the substrate, therefore making it possible to estimate the substrate induced changes of the thermal expansion coefficient and the temperature dependence of the electrical resistivity.[40]

At slightly higher frequencies, the out-of-plane ZO' mode can be observed (Figure 2), which corresponds to interlayer motion along the z-axis (a layer-breathing mode). The other out-of-plane optic modes are characterised by the doubly degenerate $Z O$ branch. At the $\Gamma$-point, the interlayer coupling causes the LO and TO modes to split into two doubly-degenerate branches, both of which correspond to in-plane relative motion of atoms. With the exception of the ZA and ZO' modes, all the frequency branches have symmetry-imposed degeneracy at the zone-center (Figure 1). 


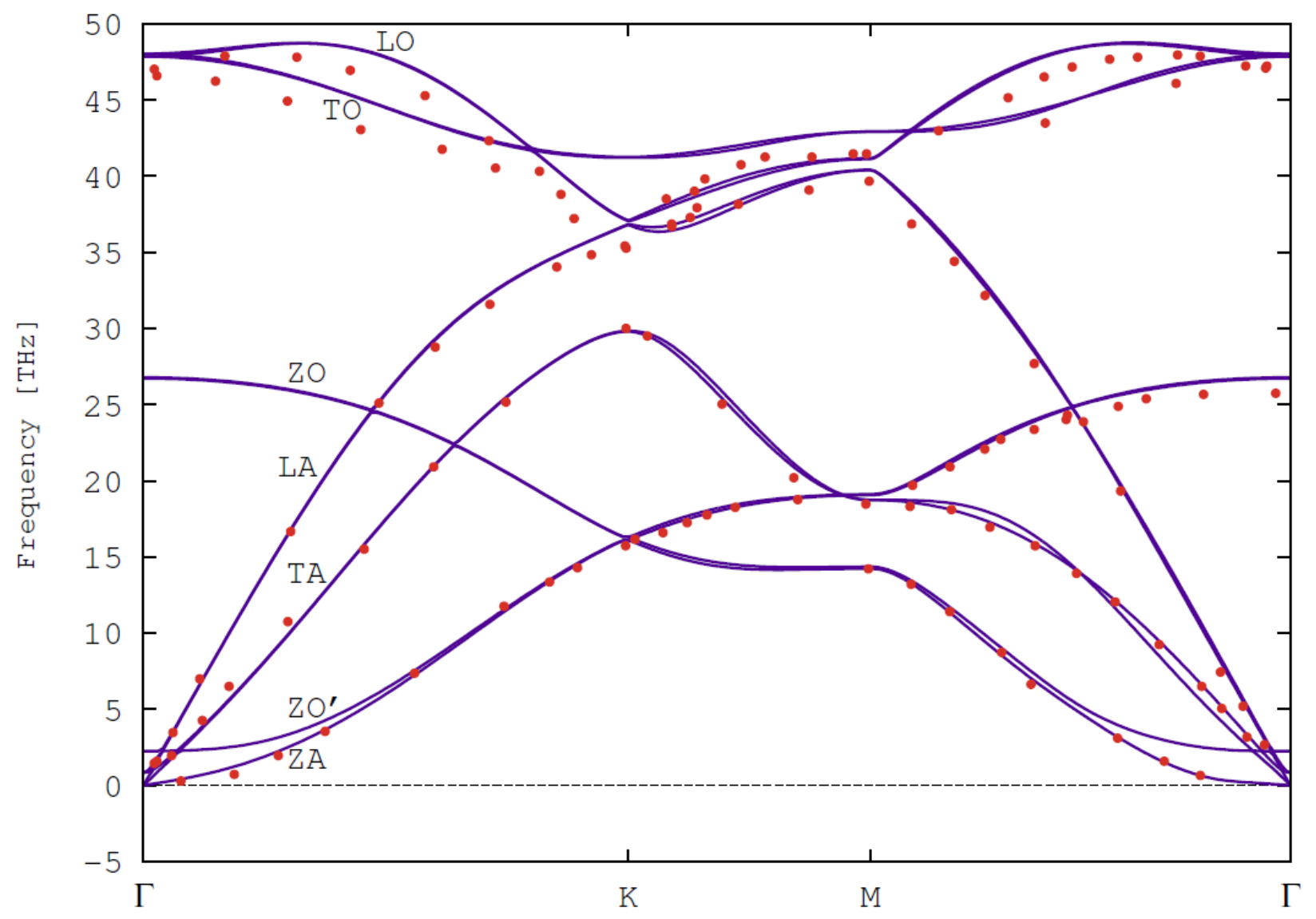

Figure 1. Phonon dispersion of $A B$-stacked bilayer graphene computed within the harmonic approximation (solid purple line). The unit cell contains four carbon atoms, leading to three acoustic (A) and nine optical $(\mathrm{O})$ phonon branches. The calculated dispersions are compared to the in-plane phonon dispersion of graphite obtained from inelastic X-ray scattering (red dots). The phonon branches are marked with the labels assigned to the $\Gamma$-point phonons. Is there a point in negative numbers in $Y$ ?

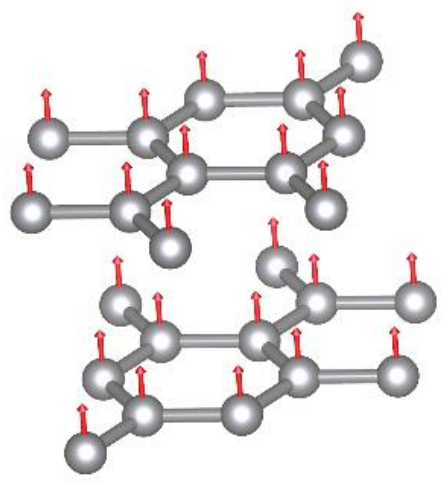

ZA

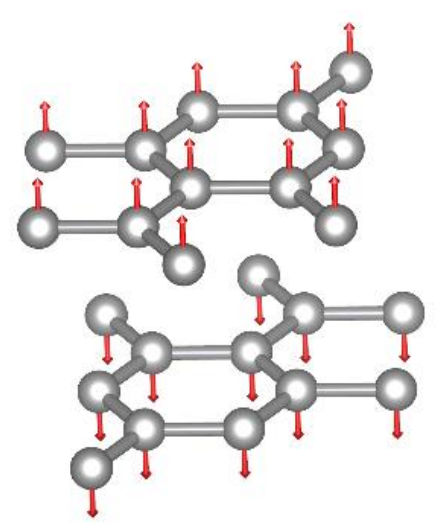

ZO' 


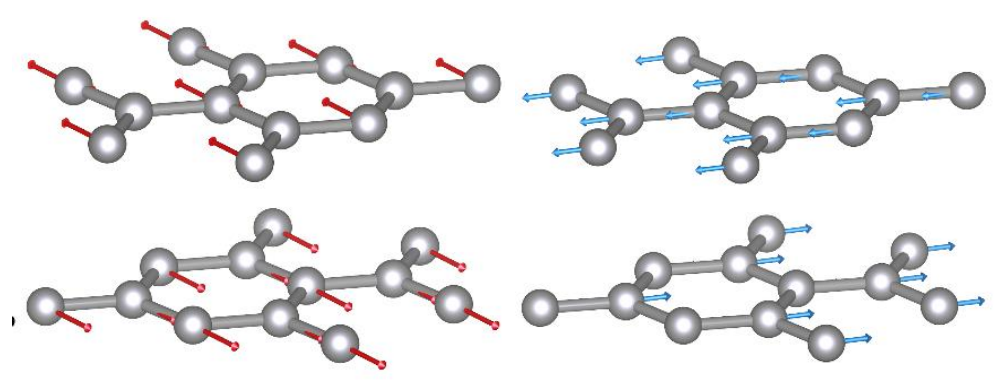

TA

Figure 2. Eigenvectors corresponding to the vibrations in AB-stacked bilayer graphene. ZA (top left) and ZO' (top right) correspond to the out-of-plane vibrations, while TA (bottom) denotes the degenerate in-plane transverse-acoustic modes.

To correctly describe the dispersion of the LO and the in-plane TO phonon branches near the $\Gamma$ - and $\mathrm{K}$ - points, it is important to consider the renormalization of the phonon energies, associated with a process in which a phonon can create an electron-hole pair.[37] These very consideration will need to be taken into account whether graphene is studied within materials science or in biochemistry. In order to understand the effects of the electron-phonon coupling one should resort to methodologies that go beyond the framework of the Born-Oppenheimer and density-functional approximations. One of the effects of the coupling interaction is the emergence of the Kohn anomaly,[41] causing the softening of certain $\Gamma$ - and K-point phonons, leading to a discontinuity in its derivative. This effect can be caused by the strong inhomogeneities in the screening of lattice vibrations by the conduction electrons. It is shown that Kohn phonon vibrations cause the Fermi level oscillations and therefore a tunable band gap opening.[42] For functionalised graphene (with group-IV elements and different functional groups, such as $\mathrm{F}, \mathrm{Cl}, \mathrm{H}_{3} \mathrm{C}$-, and $\mathrm{H}_{2} \mathrm{~N}-$ ) the combination of an out-of-plane symmetrybreaking defect and a soft infrared-active phonon mode induces a large out-of-plane piezoelectric response into functionalized graphene.[43] It is therefore expected that the emergence of the Kohn anomaly on gated-graphene systems, will also affect the piezoelectric impulses, mentioned above, which are induced by the sliding and rotation of the adjacent graphene layers.

Regenerative medicine is currently a field of intensive interest, and thus the study of biodegradable and biocompatible materials with piezoelectric effects, such as graphene-type materials, is important. Not only can piezoelectric biomaterials be applied for bone and cartilage regeneration,[44] but also these effects have other biological applications, namely for diagnostic ultrasonography,[45] immunological biosensors[46] and pulmonary drug delivery system.[47] In fact piezoelectric effects may occur naturally in the tissues, playing a significant role in regulating and stimulating the continuous stress-induced modifications of the tissue (i.e. collagen).[48]

\subsection{Coherent or resonance spectroscopy}

A complete characterisation of the biomaterials includes, not only the study between bulk and nanosized structures as mentioned above, but also the study of the most common and abundant 
impurities and chemical defects found in these systems, i.e. hydrogen impurities and native defects (i.e. vacancies), since these alter the chemical properties of interest. The following sections will therefore highlight two major resonance spectroscopy techniques with interest for biomedical applications, focusing on the analysis of hydrogen impurities and vacancies (small voids) of biomaterials.

One such experimental technique is Muon Spin Spectroscopy ( $\mu S R$ ), a well-demonstrated technique to study several fields of sciences, which include atomic-level studies in condensed matter, molecular, chemical, biological, geological and engineering materials. $\mu S R$ enables direct measurements of intrinsic electron spin relaxation rates in spin based electronics with relevancy for several applications, including biotechnology.[49]

In biosciences, $\mu S R$ has been applied for the imaging of human brain to study new brain functions.[50] Due to the sensitive nature of the muon as a magnetic probe[51] and the selective positioning nature of the positive muon (light proton with shot lifetime) at negatively charged sites, muon spectroscopy can detect and identify the magnetism of blood cells, namely hemoglobin (nonmagnetic oxy-hemoglobin and magnetic deoxy-hemoglobin and met-hemoglobin).[50] The advantage of using such a technique as compared to magnetic resonance imaging (MRI), or positron-emission tomography (PET), is that $\mu \mathrm{SR}$ can detect the function of the brain more efficiently (deeper probing, smaller volume, and short time-scale measurements) without the need of applying high magnetic fields. Moreover muon spectroscopy can provide new aspects of the oxygenation reactions of hemoglobin since it can detect triplet excited states of oxy-hemoglobin.[50]

Implanted muons can sometimes bind to an electron to form a light isotope of hydrogen called muonium. By analysing the muonium behaviour inside a material one can learn about proton and hydrogen behaviour, which is important since the chemical and structural properties of the biomaterial will be affected.

Another technique of interest is Positron Annihilation Lifetime Spectroscopy, mostly employed in material sciences, and can provide information regarding atomic and molecular level free-volume and void sizes, molecular bonding, structures at depth-layers, phase transitions.[52] However, together with Positron Emission Tomography (PET) imaging, Positron Lifetime Spectroscopy can serve as a multi-purpose detector and be applied for medical diagnostics, for example in vivo imaging of cell morphology,[53] allowing to distinguish changes in the biomechanical parameters between normal and abnormal cells (i.e., cancer cells). Marco Difficult to cite E. Kubicz, Potential for biomedical applications of Positron Annihilation Lifetime Spectroscopy (PALS), arXiv:1810.02731 [physics.med-ph] (2018). Estelina: same as 19. Marco: my 19 is different from yours... tell me the title

Depending on the application of interest, and due to the versatility of Positron Lifetime Spectroscopy, this technique can serve in several fields with biotechnical applications (drug delivery formulations, drug encapsulation, and biocompatible used in medical products); macromolecular and membrane 
study (macromolecular cavities, membranes, and conformational states affected by hydration, temperature, UV irradiation); biological tissue research.[52]

\subsubsection{Muon Spin Rotation/Relaxation/Resonance Spectroscopy: Hydrogen Impurities}

Impurities and/or dopants may appear in materials from initial growth conditions to aging of fully developed devices,[54] [55] affecting the device properties and reliability. Among such defects are impurities, such as hydrogen species that can be unintentionally incorporated during the growth environment.[54] [56] Depending on the respective behaviour, Hydrogen can be amphoteric, where it counteracts the electric conductivity by passivating defective levels or, it can enable a donor level close to the conduction band, thus inducing $n$-type conductivity.[54] Hydrogen may indeed influence the electrical, magnetic, optical and thermal properties of the host,[54] thus altering the respective properties and hence functionality. In graphene samples a variety of functional groups can be bound to the carbon network, however the most common chemisorbed specie is found to be the atomic Hydrogen. Hydrogen impurities can generate localized spin states causing spin-half paramagnetism.[57] [58]

Due to computational simplicity and efficiency, it is still ground-state electronic structure DFT calculations that are mostly applied to obtain several properties and extrapolate to provide insights to experimental spectroscopy data. Examples are studies of defect chemistry of solids to obtain defect formation energies and electronic configurations, which ultimately describe the electrical behaviour. The position of defect levels within the band-gap can also be examined through density of state calculations, which may provide insights if the defect level is located deep in the band-gap (amphoteric) or if it is a shallow defect and therefore being resonant with the conduction states and positioned above the conduction band minimum.[56]

First-principles studies of Hydrogen in a wide range of oxides have been achieved[56] by employing mostly (semi-)local functionals within Density Functional Theory (DFT). However, it is well known that these functionals provide poor accuracy of the solids band-gaps, and are also inadequate to treat strongly correlated systems, namely $d$ - and $f$-electron systems, because the incomplete cancellation of the Coulomb self-interaction favours delocalisation.[56] Non-local hybrid functionals, have received increased interest providing reasonably good solutions for the band-gap problem.[59] This is owed to the mixing of a fraction of the GGA exchange-energy with the exact Hartree-Fock exchange. These functionals also have the advantage of being able to partially correct the selfinteraction error, providing reasonably good descriptions for the electronic structure of the $f$-electron systems. Another approach that can correct the severe self-interaction error of the (semi)-local functionals is to introduce a local Hubbard-U potential, characterized by the on-site Coulomb repulsion among the localised electrons. If the Hubbard correction is applied to spin-polarized GGA calculations, good agreement for lattice parameters and band gap can be achieved with a small value of U.[56] 
GW is another alternative to DFT for calculating the electronic properties of materials from firstprinciples, and can improve on the band-gaps of insulators and semiconductors, hence correcting the systematic DFT underestimation. Most computationally affordable GW calculations employ the LDA eigenfunctions, as the starting point, to generate the self-energy $\left(G_{0} W_{0}\right)$ from the LDA polarisation (or dielectric constant) one obtains the screened interaction, $W_{0 .}$ [26] When employing $\mathrm{G}_{0} \mathrm{~W}_{0}$ it has been demonstrated that the fundamental band-gaps in $\mathrm{sp}^{3}$ covalent materials show an improvement over LDA.[60] In spite of this, the one-shot GW band-gaps are still underestimated, when compared to experimental, even for weakly correlated semiconductors.[61] One-shot GW approaches are rather unsatisfactory, since the QP levels are closely related to the quality of the ground-state wave-functions (DFT exchange-correlation functional).

Quasiparticle self-consistent GW (QSGW) applies a Hamiltonian that is found by optimisation and therefore it is expected that calculations will predict more reliable ground- and excited-states properties for a large number of weakly and moderately correlated materials.[61] Calculations result in reliable quasi-particle (QP) levels for a wide range of materials: not only in the description of the fundamental gaps in semiconductors but also for the majority of the energy levels. Even in strongly correlated $d$ - and $f$-electron systems, errors are somewhat larger than experimental evidences, but these are still systematic.[61] Despite the increased accuracy of QSGW, this method tends to slightly overestimate semiconductor band-gaps and underestimate dielectric constants.[60] The reason for this is because $W$ does not include electron-hole correlation; the inclusion of the correlation energy would reduce the pair excitation energy in its intermediate states.[60] [61]

Therefore and in order to obtain a more precise position of the defect levels inside the band-gap, care has to be taken to employ the correct functional or methodology. Since a hybrid functional, or even (QS)GW will increase the band-gap width, the position of the defect levels will be more consistent and more reliable to compare to experimental data. Examples concern the case of $\mathrm{Y}_{2} \mathrm{O}_{3}$,[62] which is a promising material for biological imaging applications. [63] [64] Calculations have been performed with the hybrid non-local functional (HSE06). Results demonstrated the existence of metastable minimum-energy sites of which amphoteric behaviour was found for Hydrogen after considering the lowest-energy structures for each charge state. For all neutral and negative configurations, localised defect levels were found inside the gap. The calculated acceptor transition level is observed to be near midgap, and is consistent with experimental data.[62] However, for the case of lanthanide oxides, relevant for applications such as magnetic resonance imaging, X-ray computed tomography and fluorescence imaging,[65] the $f$-states enter into play. It therefore becomes difficult to perform many-body perturbation calculations, or even non-local calculations within DFT. Therefore DFT $+U$ can aid in localising the correlated $f$-states of the metal, and hence increase the band-gap width to slightly higher values when compared to the performance of (semi)local functionals. The DFT+U approach is computationally as efficient as conventional DFT, and is well suited for defect studies, which require a large number of atoms, and has also proved reliable in recent studies of defects in $f$-electron solids. Examples of this approach can be found in studies 
of Hydrogen impurities on $\mathrm{Lu}_{3} \mathrm{O}_{3}$ by employing the effective Hubbard-type potential to treat the onsite $4 f$-electron correlations (50). By considering the lowest-energy configurations to obtain the charge-transitions, results predict that Hydrogen is an amphoteric impurity in $\mathrm{Lu}_{2} \mathrm{O}_{3}$, in accordance to experimental results.[56]

Several experimental techniques exist that may extend the search for new information regarding the Hydrogen impurities. These include, electron paramagnetic resonance (EPR), where Hydrogen is studied in the paramagnetic state or under non-equilibrium conditions (because interstitial Hydrogen is not stable in the neutral charge state). Such a technique provides insights into redox biochemistry research, due to its ability to distinguish and quantify the hydroxyl radicals and hydrogen atoms.[66] Nuclear magnetic resonance (NMR) is yet another method that can gather information about the bonding configurations of Hydrogen and which is widely applied to biomedical and pharmaceutical research.

In spite of the advantages, the use of microscopic techniques, like EPR or infrared (IR) vibrational spectroscopy, show some drawbacks when gathering microscopic information about Hydrogen configurations and electric defect levels, since these are limited to systems where Hydrogen is usually present in high concentrations.

Moreover, since isolated Hydrogen impurities are very difficult to study experimentally, because they possess high mobilities and thus tend to pair with other defects, [55] [67] muon spin resonance is a relevant technique to study Hydrogen in biomaterials, [68] providing very similar results to EPR. It is an experimental technique performed on muonium, which is an electron bound to a positive muon, being a pseudo-isotope of Hydrogen with similar chemical behaviour. From this spectroscopy technique it is possible to exploit the muon spin rotation, relaxation and resonance.[62] The short lifetime $(2.2 \mu \mathrm{s})$ of muonium reflects short time-scale measurements under non-equilibrium conditions, thus favouring observation of the isolated defect centers, modeling exactly those centers that are responsible for the electrical activity of Hydrogen.[62] Although the muon is only one ninth the mass of the proton, it is still possible to compare the respective properties to those of Hydrogen in a wide range of materials. These properties include electronic structure, thermal stability and charge-state transitions,[62] often in a parallel approach with ab-initio studies.

Muonium spectroscopy has yet another advantage since this technique can provide detailed information about the electronic structure of muonium/Hydrogen through the hyperfine interaction (refers to small shifts and splittings in the energy levels due to the interaction between nuclei spins and electron density).[69] Such information is difficult to obtain for isolated Hydrogen from any other technique. The calculations of the hyperfine tensors proved useful information in characterising the neutral impurity centers, thus allowing a direct comparison with the experimentally obtained hyperfine constants measured by $\mu \mathrm{SR}$.[56] It is important to be aware that when providing ab-initio results for comparison of the hyperfine tensors at the atomic sites, one needs to be aware of the exchange-correlation functional employed to compute the hyperfine tensor. It is well known that a pure PBE approach tends to delocalise the spin density of defect centers.[69] Hybrid functionals 
provide reasonably good results in comparison to the experimental data, since the defect centers will result in more localised centers. For the case of the studies performed on $\mathrm{Lu}_{2} \mathrm{O}_{3}$, calculations of hyperfine constants for the neutral interstitial configurations show a isotropic hyperfine interaction with two distinct values of $926 \mathrm{MHz}$ and $1061 \mathrm{MHz}$ for the Fermi-contact term which correspond to two interstitial positions of hydrogen in the lattice. These high values are consistent with the muonium spectroscopy measurements, which also reveal a strongly isotropic hyperfine signature for the neutral muonium fraction with a slightly larger magnitude of $1130 \mathrm{MHz}$.[56]

From a theoretical point-of-view, through DFT calculations, the higher energy configurations (metastable states) that may exist for the different Hydrogen charges, should be considered in order to compare with $\mu S R$. This will allow defining the charge transition levels for each structural configuration that Hydrogen can adopt in a system. This procedure is more realistic when comparing to non-equilibrium muonium measurements since, and due to the short life-time of muonium $(\sim 2.2$ $\mu \mathrm{s})$, it is not possible to obtain full equilibrium measurements. Instead higher energy metastable states can also be accessed together with their acceptor and donor levels individually.

The material thermodynamics depends on the formation energies of different types of defects and therefore the stable charge states and their transitions can be determined from this quantity.[70] For the case of the Hydrogen impurity, the formation energies are hence evaluated to obtain the properties of interstitial Hydrogen in the host material and this is defined as being the energy required to incorporate the impurity in three charge states $\left(\mathrm{H}^{-}, \mathrm{H}^{0}, \mathrm{H}^{+}\right)$in the host lattice.[67] [69]

Having determined an adequate number of minimum-energy Hydrogen configurations it is also important to identify representative pathways for the impurity migration that connect initial and final ionic configurations. This type of study can be carried out by employing the nudge-elastic band (NEB) method.[71] From this method it is possible to compare the proton/muon sites and the activation energies between equivalent sites.[72]

Polarised muons have proved to be reliable and sensitive probes of local magnetic fields in matter and an important tool for the investigation of Hydrogen reactions in low electron density materials (i.e. graphene). In defective graphene, muon spectroscopy aids in answering two debated questions: the possible onset of magnetism and the interaction with Hydrogen.

It is possible to observe clear muon spin precession in graphene samples, contrary to the behavior of graphite, and this has been demonstrated to originate from a localised muon-Hydrogen nuclear dipolar interactions, rather than to a hyperfine interaction with magnetic electrons, and reveals the formation of an extremely stable $\mathrm{CHMu}$ states (analogous to $\mathrm{CH}_{2}$ ). These results rule out the formation of magnetism in chemically synthesized graphene samples. Moreover, in $\mu S R$ spectroscopy electronic states of edges and defect sites have revealed radically different signals with respect to the standard case of graphite, or even of the pristine graphene plane.[58]

\subsubsection{Positron Lifetime Spectroscopy: Vacancies}


Vacancies are an example of one of the most common defects found in graphene and can be induced by proton irradiation.[58] When a $C$ atom is removed from the lattice, breaking of the symmetry of the system induces a band-gap and the three $\sigma$-dangling $\mathrm{sp}^{2}$ bonds introduce localised states in the mid-gap, which split due to the crystal field effects and a Jahn-Teller distortion.[73] While two of the electrons bind to each other to form a pentagonal ring, the third dangling bond becomes a paramagnetic center. One impurity may induce magnetisation in the graphene lattice due to formation of spin-polarised localised states. However, the presence of several vacancies may affect the magnetisation of the system due to correlation of the impurity position. Also, since the presence of only one vacancy allows symmetry breaking of the lattice (inducing a band-gap), two identical vacancies, located at the same sub-lattice, will increase asymmetry, therefore increasing the gap width. Therefore breaking of the symmetry may define the magnetic properties of the lattice, allowing for the defects to interact with each other as being ferromagnetic or antiferromagnetic, depending on the extension of the asymmetry. The Fermi level of graphene is quite sensitive to perturbation, and alterations may occur due to the mismatch and subsequent strain at surfaces or interfaces or due to impurities at the interface of graphene.[73] not sure which interface one refers to- spell it out.

It is therefore important to investigate the defects or chemical impurities at the interface of the multilayer or single layer graphene with biosurfaces. The choice of the substrate on which graphene is deposited plays an important role in the electronic, magnetic and chemical properties of the film. It is relevant to employ a non-destructive probe, which can investigate graphene over layers on various substrates without extensive sample preparation or probe modification of its chemical or physical properties.

A well-developed non-destructive experimental technique suited in probing point defects, mainly vacancy-type defects, is positron annihilation spectroscopy. By measuring positron lifetimes and momentum distributions during the annihilation process, it is possible to obtain information on the electronic structure in solids, mainly the open volumes and the chemical environments of the defects, i.e vacancy-type defects.[57] The bound state of a positron and an electron is analogous to a Hydrogen atom, where the proton is replaced by the positron. The annihilation radiation contains information on the electron momentum distribution at the annihilation site due to conservation of momentum during the annihilation process. Though there is the tendency for the electron and positron to annihilate when close to each other, under certain conditions these can form a bound state before annihilation occurs. If the thermalised positron is in the vicinity of an electron, the two charged particles will be attracted by their electrostatic charges instead of being annihilated, hence forming a positronium.[74]

From a computational point of view, it is possible to employ a two-component generalization of the Density Functional Theory (TCDFT), to probe the positron state and annihilation characteristics. The positron-electron correlation-energy functional is usually calculated on the limit of the vanishing positron density. For a delocalised positron the respective density is small at every point of the lattice, 
not influencing the electronic structure, and hence the electronic structure of the perfect lattice is solved separately from the positron density. When the positron and electron densities are known, then the positron annihilation rate can be calculated.[70] [75]

When a positron is localised at a lattice defect, one has to take into account the fact that the positron attracts electrons, and the average electron density increases near the defect. The short-range screening effects have hence to be taken into account by correlation functionals which depend on both the electron and the positron densities. Nevertheless, in most applications for positron states at defects, the full scheme is simplified by using the same procedure as that for delocalised positrons. This means that the zero-positron-density correlation energy and enhancement factors are used. The simplified scheme can be well justified by arguing that the positron, and the respective screening cloud, forms a neutral quasiparticle that enters the system without changing the average electron density. On the other hand, the two-component calculations also support the use of a conventional schemes within the LDA or GGA approximations.[75]

In order to investigate two dimensional materials like graphene, it is essentially important to trap the positrons on the surface state. If positrons are implanted at energies less than $10 \mathrm{eV}$ then it is possible to obtain the relevant information from the top atomic layers of the nanoscaled sample as there is greater probability for trapping the positrons into the surface state.[76]

Recently, measurements using a low energy positron beam have been reported and applied for the study of multilayer graphene adsorbed on a polycrystalline Cu sample.[76] The efficient trapping of positrons on the surface state of graphene at kinetic energies as low as $2 \mathrm{eV}$ has been used to obtain the respective Doppler broadened spectra. This is the first ever measurement of graphene thin-films (2-3 nm of thickness) adsorbed on a substrate with a depth resolved Doppler broadening spectroscopy at positron kinetic energies below $10 \mathrm{eV}$, to have been reported so far. These findings open the possibility of employing positron beam spectroscopy to characterise defects and chemical structure in nanometre $2 \mathrm{D}$ materials. 


\section{Computational Techniques for Biocompatible Materials}

Biological reactions are mainly induced by interactions between the surrounding environment and the surfaces of the materials. Thus, the key parameter to focus on in order to reduce or at least control the influence of a material at the bio-interface is its surface. Biological reactions are not always well understood and this chapter will describe how computational analysis can provide excellent tools to explain the interactions of materials surfaces with biological media. We will explain the scientific questions and focus on the computational analytical techniques that can measure these properties at the interfaces. In a first part, we will discuss which properties of the surface are crucial. We will discuss the importance of the chemistry or the roughness biocompatible materials and present the computational techniques that can be used to investigate these surface characteristics. The biological behavior of surfaces is controlled in a large part by interactions between a specific surface (with specific properties as will be discussed in the first part of the chapter) and different (bio)molecules present in the surrounding media. This will be the subject of the second part, where techniques able to study and predict surface interactions with bio-molecules such as polymers or proteins will be presented. The last part will finally discuss the importance of size in the case of nanoparticles bio-interactions, which has a large influence on colloidal stability, the formation of the so-called corona of proteins or other molecules or on the formation of micelles.

\subsection{An Overview of Surface Properties}

As generally most interactions between biocompatible materials and any biological system happen at the surface of the material, the surface properties are of paramount importance. From an experimental point of view, despite accurate control of synthesis processes, understanding the phenomena at the interface is often neglected leading to biased observations and conclusions. The types, number and arrangement of chemical groups at the surface will determine what species adsorb[77] [78] [79] [80] [81] and what surface reactions can take place.[82] [83] In this section, we will discuss therefore how computational solid state techniques can help define those properties of the surfaces likely to be exploited in biomedicine. We will discuss the equilibrium properties of pure materials and how to study them using different computational techniques. Then we will focus on the influence of defects, the environment and non-equilibrium structural features, such as growth features.

\subsubsection{Amorphous Solids}

Surfaces cost energy. The lowest energy (equilibrium) arrangement of the atoms is disturbed, leading to a higher energy of the atoms/molecules at the surface. The energy contribution of the surface is more important for small structures than for large, due to the different scaling of the surface $\left(d^{2}\right)$ and the volume $\left(d^{3}\right)$ with the typical dimension $d$. Therefore the shape of small (typically submicrometric) structures are often dominated by the minimization of the surface energy. For amorphous solids, where the surface energy is independent of the orientation of the surface, this typically means a spherical shape, optimizing the surface to volume ratio. For larger structures of 
amorphous solids, where the contribution of the surface energy becomes minimal, the chemical groups present at the surface are those that will minimize surface energy.

\subsubsection{Ionic and Covalent Crystals}

For the crystalline phases the energy of an interface depends on the orientation with respect to the crystalline lattice. The reason for this is that the orientation of the interface will determine the nature of "bonds" present in the original crystal structure that will be broken in forming the surface. The dependence of the interfacial energy on the interface orientation with respect to the crystal lattice means that the shape, minimizing the total interfacial energy of a finite sized crystal, is no longer a sphere. Wulff developed a general method to predict the equilibrium morphology of a finite sized crystal based on the interfacial energy per unit area $\gamma(\phi, \theta)$ as a function of the orientation.[84] In fact Wulff showed that, in order to minimize the overall interfacial energy, the distance of an interface from the center of mass should be proportional to $\gamma(\phi, \theta)$ (Figure 1). The Wulff construction demands knowledge of the interfacial energy with respect to the interface orientation. However $\gamma(\phi, \theta)$ is not easily accessible with experimental methods.

a)
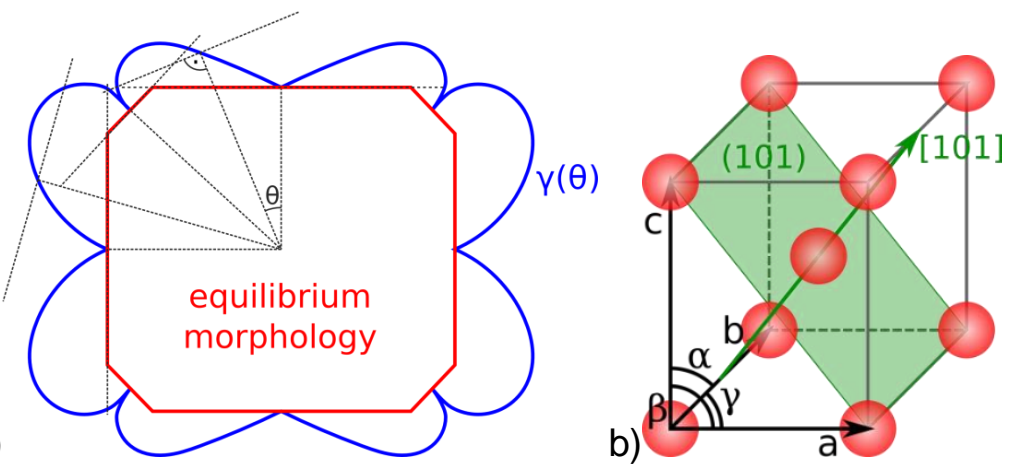

Figure 1. a) Schematic illustration of the Wulff construction in 2D: The interfacial energy curve is shown in blue, the perpendicular lines to the $\Theta$-direction at the position of $\gamma(\phi)$ used for the Wulff construction in black and the resulting equilibrium morphology corresponding to the inner envelope to all perpendicular lines in red. b) Schematic illustration of the cell parameters $\vec{a}, \vec{b}, \vec{c}$ and $\alpha, \beta, \gamma$. Also shown is the (101) surface with the surface normal (101), where the numbers in parenthesis are the Miller indices indicating the orientation of the plane. Illustrations adapted from (11). These are generic images, what about changing them slightly so that there is no need of citation and no probs with copyright.

In the case of ionic crystals, the low energy interfaces are usually parallel to a crystal plane (h k I), where $\mathrm{h}, \mathrm{k}$ and $\mathrm{I}$ are the Miller indices of the plane indicating its orientation of the plane with respect to the crystal lattice (Figure $1 \mathrm{~b}$ ). For a crystalline plane, $h, k$ and I are inversely proportional to the intercept of the plane with the vectors of the crystal lattice and for most lattices are equivalent to the indices of the surface normal. The continuous function $\gamma(\phi, \theta)$ can thus be reduced to a certain number of surface energies of different crystallographic planes (h k I). Atomistic simulation techniques can be used to determine the enthalpic contribution to the surface energy of different 
atomistic planes, taking into account the relaxation of the position of the atoms at the surface. This can be done either using DFT[85] [86] [87] or classical atomistic force fields (potentials).[88] [89] The energy of a surface is calculated by comparing the energy of the relaxed surface to that of the bulk crystal structure. Usually crystals are represented by periodic boundary conditions, or in other words by a small part of the crystal that is thought to repeat in all three dimensions to build up a much larger bulk structure. However in direction of the surface normal, the periodicity of the crystal is disrupted and periodic boundary conditions can no longer be applied in this direction. There are two main methods to deal with this 1D lack of periodicity. (1) If an energy minimization or structural relaxation technique is used to find the lowest enthalpy structure, the bulk structure is often represented by a region with pre-relaxed, fixed atomic positions adjacent to the surface layer which is allowed to relax.[88] (2) If molecular dynamic calculations are performed instead, the surface is often represented by a slab of material, terminated on both sides by a surface.[90] While the surface energy $\gamma(\phi, \theta)$ depends on the surface directions, there might be different surface terminations to take into account, resulting from the different nature of the broken bonds through the crystal at different depths, which share the same surface normal. To get the correct surface energy, the termination with the lowest energy has to be considered.[91] Additionally some surfaces might present large surface restructuration (local rearrangement of the atoms close to the surface), something that will have to be considered explicitly.[92]

\subsubsection{Metals}

So far we only discussed the enthalpic contribution to the surface energy. For ionic crystals this is generally by far the dominant contribution to the surface energy, justifying the estimation of the surface energy directly from energy minimization or molecular dynamics. For metals however the surface free energy is often important. Consequently different methods have been developed to calculate the surface free energies of metals: One being a thermodynamic integration technique[93] [94] [95] and the other based on the characterization of fluctuations at the surface.[96] [97] [98] [99] Again the aim of these techniques is to determine the anisotropy of the surface energy to be able to predict growth, morphology and the surface properties of the final material.[100]

\subsubsection{Influence of the Environment}

The environments (melt, solution, gas phase) around the solid both during the formation/synthesis and in the end application also have a significant influence on the surface properties. While the solid is growing, the interactions with the species from e.g. a melt or a solution can change the relative surface energies and consequently the equilibrium shape.[78] [90] The same if the material is surrounded by an aqueous environment. For calcium hydroxide, the stronger interaction of water with the higher energy surfaces compared the low energy basal plane is likely to be the reason why particles precipitated from pure, supersaturated $\mathrm{Ca}(\mathrm{OH})_{2}$ solutions do have the shape of hexagonal platelets that is observed for naturally occurring minerals.[90] As these interactions are generally transient and changing constantly, as well as the fact that liquids in contact with surfaces generally show density variations perpendicular to the surface in the length scale of $0.5-1 \mathrm{~nm},[80]$ [101] only 
simplified systems with the adsorption of few molecules are generally studied with DFT or ab-initio methods,[82] [86] [102] [103] while for the study of the larger scale systems, the preferred method being classical atomistic simulations.[90] [101] If the surface is reacting with species from the environment, on the other hand DFT is more frequently used in these studies, as chemical reactions are more easily described by DFT. For oxides for example, DFT in combination with thermodynamic calculations allow the prediction of the conditions, under which surfaces are likely to be hydrogenated or hydroxylated,[82] [85] [86] or it can be used to estimate the acidity of surface groups.[104] Especially for metals, surface reactions, such as oxidation, can lead to the formation of surface layers, which again can be studied using atomistic methods.[105]
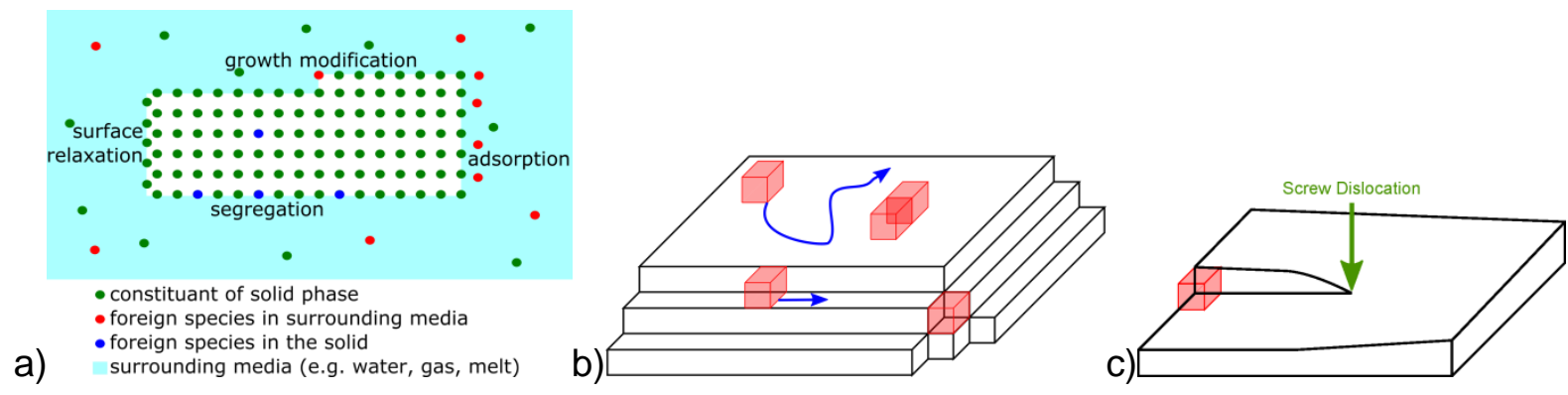

Figure 2. Schematic illustration of possible influences on the morphology of solids a), different types of surface sites and surface diffusion b) and defect growth mechanisms c). Adapted from (11).

Defects and species in solution can have a higher or lower affinity to the interface region than to the bulk (Erreur! Source du renvoi introuvable.a), leading to a concentration gradient within the material normal to the surface. The energetics of this segregation of defects or adsorption of surrounding species can be estimated by atomistic simulations.[78] [81] [106] The easiest is to calculate the adsorption or segregation enthalpy, by comparing the energy of the bulk and the interface positions. However care has to be taken to consider all relevant positions at the interface. To estimate the free energy difference more advanced methods, such as nudged elastic band, metadynamics or other free energy methods are needed.[80] [81] An additional complexity is the distribution and equilibrium concentration of defects. For the estimation of this, additional methods are needed. In the case of weak surface adsorption from solution, direct molecular dynamics simulation of the system over long enough times allows the estimation of the distribution of the species in solution.[107] However due to the limited possible size and length scale of the simulations, this approach is limited to systems with weak adsorption and fast dynamics as well as to large concentrations ( $>1 \mathrm{~mol} / \mathrm{L}$ ). This is of particular interest for biological interactions as biological fluids interacting with materials are usually highly concentrated. For other systems, either additional thermodynamic calculations[108] or grand canonical (or Metropolis) Monte Carlo calculations[109] are needed. Within this context single surfaces are also often extended to porous media. A special case arises, when the species considered are charged which is the norm in biological media. In this case the adsorption/segregation will lead to a spatial separation of charges normal to the interface. 
In the case of solids one generally speaks of a space charge region, in the case of adsorption from a solution of an electrical double layer. For the latter a charge separation can also be caused by surface reactions such as acid/base reactions of surface groups. For both segregation[110] [111] and adsorption[112] [113] the distribution of the charges is often calculated using continuum methods (that is approximating the distribution of discrete charges by a continuous charge distribution), often using the results of atomistic methods However in the case of the electrical double layer, Monte Carlo methods are also frequently used.[104] [114] [115]

\subsubsection{Non-Equilibrium Effects}

In the discussion above, we assume that equilibrium properties determine the surface properties. However, for crystals growing from a solution, the prevalence of a surface is often also controlled to a larger or lesser extent by the speed of growth in the direction of the surface normal. Control of the growth is also one method to control the surface roughness which is an important parameter for cellular attachment and proliferation.[116] The main method is to grow nanostructures at the surface of the materials to provide specific properties such as anti-biofouling.[117]

In order for the crystal to grow, the atoms have to move first from the bulk solution to the interfaces. Schematically one can imagine different types of surfaces for which the adsorption process can be different: atomically flat surfaces which run parallel to a certain crystal plane, stepped surfaces which only one direction in common with a crystal plane and finally kinked surfaces that have no direction in common with any crystalline plane (Erreur ! Source du renvoi introuvable.b). An atom that adsorbs at a kink site will immediately become part of the crystal and indistinguishable from other surface sites. An atom that adsorbes at a step site still retains a certain mobility in one direction, due to the reduced number of bonds formed with the surface compared with a kink site. In order to definitely become part of the crystal it would have to associate with one or more other atoms, forming a one dimensional nucleus, terminated on both ends by a kink site that can then grow easily. Alternatively an atom adsorbed at a step site can move along the step until it finds a kink site where it can be integrated into the crystal. An atom adsorbed at a flat surface is even less strongly bound to the surface and even more mobile. It can undergo 2D surface diffusion until it either encounters a step or a kink site or other surface atoms to form a 2D layer nucleus. Generally the relative surface energies also give an indication of the relative growth rates as they are governed by similar properties: a kinked surface will clearly grow faster than a stepped and much faster than an atomically flat surface and at the same time can be expected to have a much higher surface energy. Similarly solvent or solute species adsorbing at flat, stepped or kinked surfaces preferentially will slow down growth in that direction, due to the competition of the adsorbed species with growth species, and will lower the relative surface energy. However there are a few exceptions where kinetic effects should be considered.

First of all for a growth species to adsorb, it will have to move through the electrical double layer and through the structured water layers[101] [118] and it will have to lose its hydration shell. These processes are often associated with an energetic barrier which can vary from surface to surface. 
Such effects can be studied by the same free energy methods that can be used to study the adsorption of other species from solution.[80] Additionally the changing diffusion coefficient of species in the structured water layers close to the surface[101] as well as the surface diffusion coefficient[119] can be of importance for growth. Diffusion coefficients can either be estimated form activation energies[120] or from direct molecular dynamics calculations, however especially for the latter great care has to be taken to take into account the correct time scale that allows for large scale diffusion and not to be limited to fast, local movements.[121] [122]

Growth in a certain direction can be accelerated by the presence of defects at the surface, namely screw dislocations. A screw dislocation is a line defect where, at a certain line in the crystal, the atomic planes are partially shifted by an interatomic distance parallel to the defect line. If the screw dislocation reaches a surface, step sites will be created over part of the crystal (Erreur ! Source du renvoi introuvable.c). This step can now grow in a spiral fashion without ever reaching the edge of the surface and being annihilated. This means that due to the presence of screw dislocations, a flat surface oriented parallel to an atomic plane can grow continuously without needing to periodically go through a layer nucleation step. This process is well known to influence growth[123] and to be of big importance for the growth of nanoscale structures such as nanorods or nanotubes.[124] [125] The energetics and the atomic structure and even the kinetics (using coarse grained kinetic Monte Carlo) of the growth of these dislocations can be studied using atomistic simulation.[126] [127] Finally growth can also happen by ordered attachment or aggregation of preformed nanocrystalline building blocks, especially if mediated by organic molecules,[128] a process that can also lead to the formation of screw dislocations that will promote further growth[129] and that has already been studied using atomistic simulation.[130]

\subsubsection{Example of Application}

After having discussed in detail different ways in which computational solid state methods can and have been used to understand the properties of surfaces relevant for biocompatible systems, let us look at a specific application and how the different techniques were applied for that system. A good example of application of the techniques described above is the study of $\mathrm{TiO}_{2}$ for photocatalytic water treatment. $\mathrm{TiO}_{2}$ is one of the most promising candidates for "Advanced Oxidation Processes (AOPs)", which are based on the generation of highly reactive oxygen species (i.e. $\mathrm{H}_{2} \mathrm{O}_{2}, \mathrm{OH}, \mathrm{O}_{2}^{-}$, $\mathrm{O}_{3}$ ) at the surface of particles suspended in the water via photocatalysis. These reactive species then lead to the mineralization of refractory organic compounds, water pathogens and disinfection by-products, without creating any secondary pollution. However, photocatalytic processes will improve the production of reactive oxygen species (ROS) leading to potential environmental toxicity.[131] It is crucial to have a good understanding of the surface properties to understand, predict and improve the efficiency of the surface reactions in order to decrease their potential undesired side effects with living environment. Thus computational methods, in combination with experimental techniques, have been used extensively to know which surfaces are low-energy and thus present on the particles,[132] [133] how these surfaces are modified by restructuration[134] or 
surface reactions[86] and defects[135] and finally how the photocatalytic synthesis of reactive oxygen species is happening at the different surfaces.[82] [83]

\subsection{Surface Adsorption and Surface Functionalization}

As discussed above, the properties of surfaces are of great paramount importance for biological systems and are often tailored to selectively control the materials properties. Within this context surface properties are modified by the steered adsorption or grafting of organic molecules providing additional surface functionalities. Typical examples are implants, stents or antibacterial surfaces, which are often specifically functionalized to tailor their biological interactions. For example, surface functionalizations can prevent, on different engineered surfaces (Silica-, Titanium- or polymericbased for example), the proliferation of micro-organisms such as bacteria or fungi.[136] The functionalizations can also change the charges at the surface. The surface functionalization is mainly done by adsorption of small chemical groups providing new charges or new surface groups without completely masking the material surface properties. For example, small molecules containing amino $\left(-\mathrm{NH}_{2}\right)$ and/or carboxylic $(-\mathrm{COOH})$ groups are often used to charge the surface respectively positively or negatively, or to be able to graft further functional molecules.[139] [140] Another approach consists in adsorbing large molecules such as polymers onto the material surfaces to change their biological interactions or protect the integrity of the bulk material. Polyethyleneglycol (PEG) or Polyvinylalcohol (PVA) are used to protect the surfaces of materials from biofouling to colloidally stabilize particles. In the special case of particles to be injected or added to biological systems, either in vitro or in vivo, the surface properties will determine amongst other things the cellular uptake and their biodistribution.[137] [138]

In addition to this steered or voluntary adsorption, in biological media the interaction between the surface and the media generally leads to the formation of a layer of adsorbed bio-molecules. Recently it has become clear that, to understand the final behavior of bio-interfaces, the adsorption of proteins onto the surface plays a crucial role, something that is still being studied intensively.[141] [142] [143] [144]

Thus whether steered or unsteered, the adsorption of organic molecules is a very important process for biocompatible materials. In this chapter, we will discuss different phenomena that are important for the interaction between surfaces and organic molecules and we will discuss different computational methods that can be used to study them.

\subsubsection{Influence of the functional groups of adsorbed molecules}

The organic molecules in question possess different functional groups, the molecules (polymers or proteins) can be very large and they are often charged. These three aspects are often studied with different techniques. The interaction of different functional groups with the surface are generally studied for small molecules, peptides or oligomers with either classical atomistic methods[145] [146] [147] or DFT[148] [149], (Figure 3a), although with increasing computational time, the system sizes are also increasing.[150] For surface functionalization with small molecules, such simulations, taking 
into account the charges, can be sufficient. The limitation of the size of the studied molecules does not only come from the system size, but also of the time scale of the movement and change of conformation of larger molecules, which is much larger than the $~ 100$ ns accessible with full atomistic molecular dynamics.[151] Even using advanced techniques, such as metadynamics, full atomistic simulations of the adsorption of realistically sized larger molecules on surfaces, such as many proteins and polymers, are currently not accessible. Especially if the system of interest is not only one molecule adsorbed at the surface but a more or less compact layer of adsorbed proteins/polymers, where entanglement and confinement (Erreur ! Source du renvoi introuvable.b and Figure 3c) phenomena become important.

a)
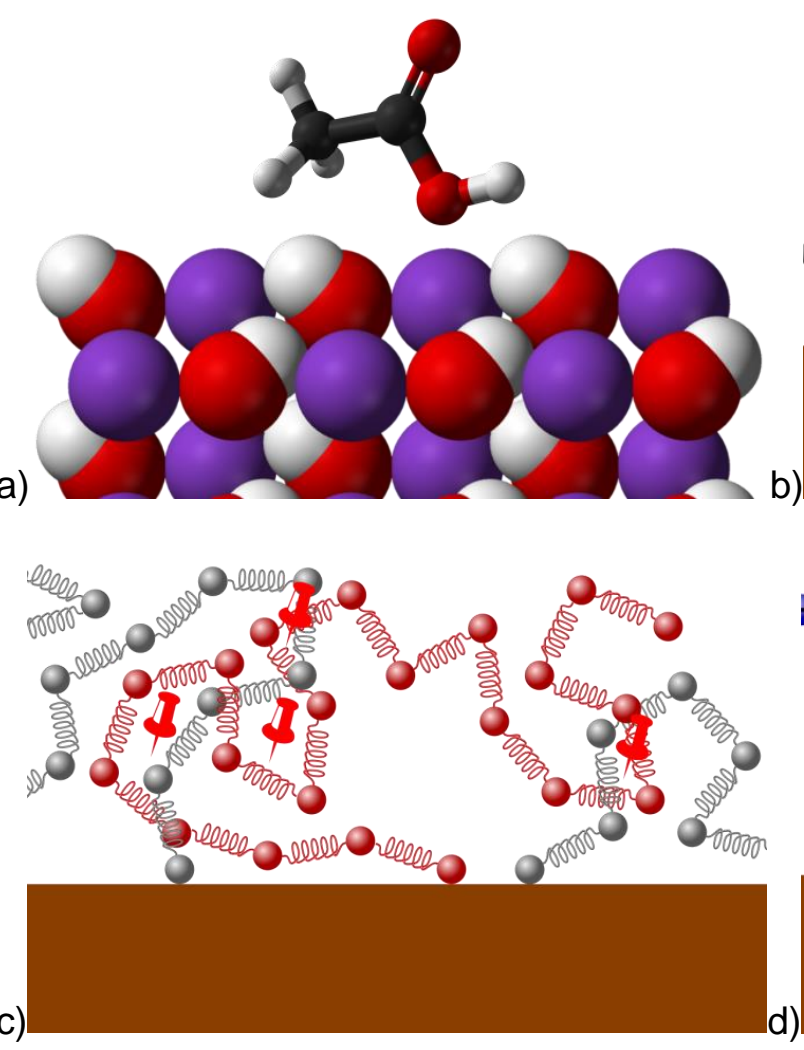
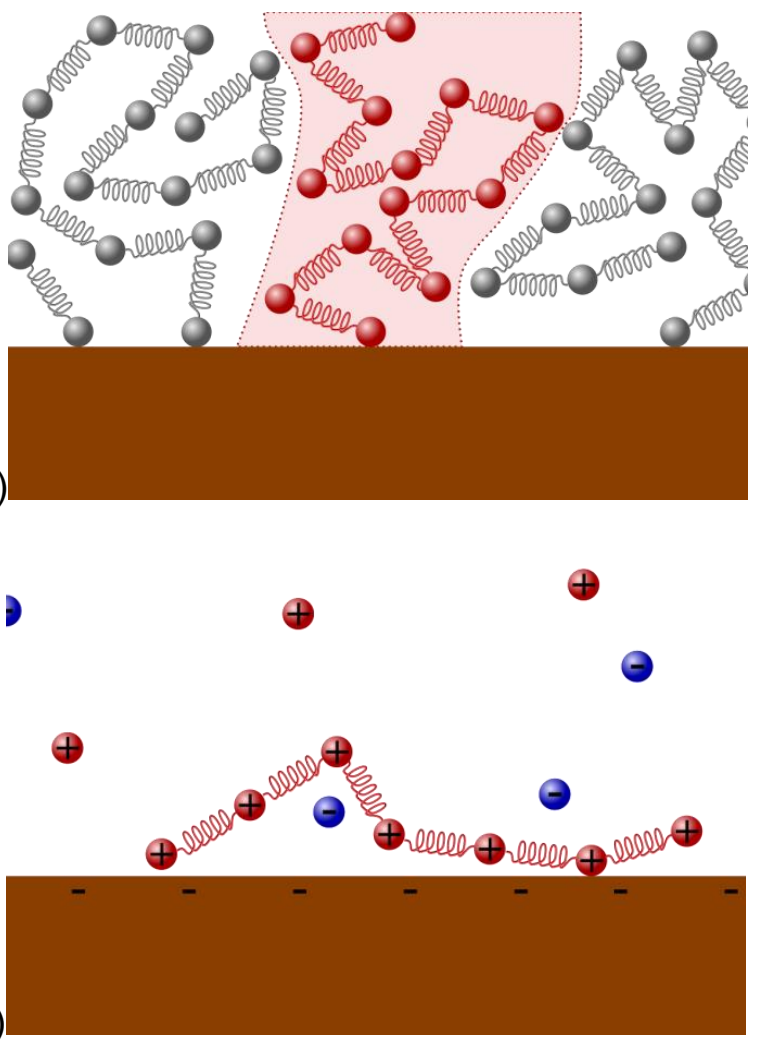

Figure 3. Different aspects of surface adsorption of organic molecules: a) atomistic interactions between surface groups and functional groups of the organic molecule; b) confinement effects due to the restricted space available within an adsorbed layer of molecules; c) entanglement effects partially pinning the different molecules and restricting its free movement; $d$ ) interdependence of the adsorption of charged molecules and the ionic distribution within an electrolyte.

\subsubsection{Influence of the conformation of adsorbed molecules}

To study the conformation of polymers or large proteins adsorbed or grafted to a surface different techniques are used. Closely related to the atomistic methods discussed above are coarse grained methods where instead of taking into account each atom, only positions of and interactions between groups of atoms are described.[152] [153] Additionally the solvent is not always considered explicitly but can be approximated by adapting the interactions between and the dynamics of the coarse 
grained entities. Approaches to adapt the dynamics of a system taking into account an implicit solvent are Brownian dynamics [154] [155] and Langevin dynamics.[156] [157] [158] The advantage of this approach is not only the fewer number of entities within the system but also the fact that, due to the larger mass and slower dynamics of the coarse grained entities, a larger timestep can be used and thus larger timescales become accessible. Such coarse grained simulations have for instance been used to study end-grafted polymers under shear flow to understand their tribological properties. [154] [157] In addition to molecular dynamics, Monte Carlo approaches are also used frequently to study the conformation of adsorbed polymers.[159] [160] Finally, due to the complexity of polymeric systems, a number of thermodynamics and mean field theories have been developed to describe the adsorption of polymers on surfaces and, at least for linear polymers[155] [161] [162] and some well-defined more complex systems[163] [164] give valuable first insight into the phenomenon governing their conformation and behavior. As an example these theories have been used to explain the mechanisms of thermoresponsive cell culture substrates.[165] [166]

\subsubsection{Influence of the charges of adsorbed molecules}

In theory both the full atomistic and coarse grained methods mentioned above take into account charges on the molecules. However a further complexity arises when looking at charged molecules adsorbing onto a charged surface within an electrolyte solution. In this case the conformation and adsorption of the organic molecules is influenced by the distribution of the charges in the electrolyte and the resulting electrical potential which in turn is influenced by the presence of the molecules. This phenomenon is most commonly studied using coarse grained Metropolis or Grand Canonical Monte Carlo methods, where the organic molecules are treated as polyelectrolytes[167] [168] or using mean field theory.

An example of field of study is the development of anti-fouling surfaces. The anti-fouling surfaces are specifically designed to avoid unwanted cellular adhesion and proliferation as well as the unspecific adsorption of biomolecules. A common strategy to render a surface non-biofouling is the formation of a hydrophilic or zwitterionic polymer-layer either through adsorption, coating, chemical grafting or the formation of a self-assembled monolayer.[169] The first theoretical studies into the effectiveness of these layers used mean field theories to study the effect of surface density and polymer length.[170] [171] Further atomistic[172] [173] and DFT calculations[174] showed the importance of the hydration layer on the polymer surface to understand the non-fouling properties, something that is consistent with experimental observations[175] [176] and significantly increased the theoretical understanding of these systems. Subsequent studies looked at the influence of the conformation and arrangement of the molecules in the non-fouling layer.[177] [178]

\subsection{The Special Case of Nanoscale Materials}

Nanotechnology is a research area which has seen its interest growing for the last twenty years. The possibility to use the singular properties of nanomaterials to solve long standing problems is becoming more and more promising. Nanoparticles (NPs) have become the most important 
components in nanotechnology. Their size (usually below 100nm) with specific physico-chemical characteristics, opens the way for the development of new solutions for catalysis, food industry, medicine or other applications.[179] [180] [181] The small size of the particles has different effects than those of the bulk solid, as already mentioned above. The physical solid state properties of a nanoscale structure can be different from that of larger structures due to nano-confinement, additionally the surface properties can be different due to the influence of the higher concentration of edges and kinks. Also the specific geometry of the surface (Figure 4) changes both the mechanisms of adsorption of large molecules and, in case of charged particles in the presence of electrolytes, the charge distribution close to the surface. Finally for any application of nanoparticles dispersed in aqueous environment such as most biological media, thus for biomedical applications in particular, the small size and change in adsorption behaviors influence the stability and colloidal interactions of nanoparticles. Thus in this chapter we will discuss the special case of nanoparticles and other nanoscale materials as well as their interaction and will present computational methods that can be used to study them, as well as their current limitations.

\subsubsection{Nanoparticles}

Many nanostructures exhibit interesting electrical, optical or magnetic properties which are directly linked to their size. These properties are either the effect of the high surface to volume ratio and the ensuing high influence of the surface (e.g. lower melting temperature, different phase diagram etc.) or due to the intermediate nature between molecule and continuous 3D solid (e.g. band structure, optical properties etc.). For the latter quantum mechanics methods (e.g. DFT) are of most interest; however, the systems cannot be treated using methods used for bulk solid-state materials such as periodic boundaries. On the other hand many of the nanostructures used are too large to be studied by DFT, thus DFT studies are generally restricted to small nanoparticles or clusters[182] [183] or to nanoparticle model structures.[184] Using classical atomistic methods, larger structures can be studied to gain insight into e.g. changes in the relative stability of different phases or other thermodynamic properties.[185] [186] Another field of study where classical atomistic methods are frequently used is the study of the edge and kink-site dominated growth and equilibrium morphologies of nanoparticles which can deviate significantly from that of large particles discussed above.[185] [187]

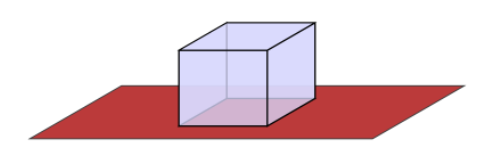

a)
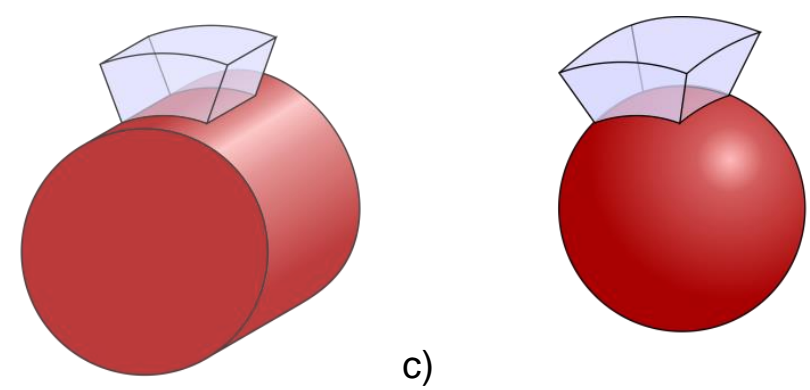

c)

Figure 4. Schematic comparison between volume available to macromolecules adsorbed on a) a planar surface (large particle), b) on a nanorod and c) on a nanoparticle. 
The small volume of nanoparticles has a significant effect on the volume available to adsorbed species and thus influences the adsorption notably of macromolecules (Erreur ! Source du renvoi introuvable.). Mean field theories on the adsorption of linear molecules can take this effect into account to some extent.[161] However for atomistic methods, already the adsorption of larger molecules on flat surfaces is a challenge, as discussed in the previous section. For nanoparticles now the systems to be studies are even larger, as periodic boundary conditions cannot be applied in the same way as for flat surfaces. Consequently in the vast majority of studies on polymer/protein adsorption on nanoparticles, coarse grained methods are applied.[156] [188] [189] [190] [191] In addition most of the time oligomers rather than larger molecules have been studied,[188] [192] [193] or the nanoparticle has been simplified.[189] [190] [194] Other coarse grained studies specifically deal with the adsorption of a single charged polyelectrolyte.[195] [196]

\subsubsection{Other nanoscale materials}

There are two related fields that do look at similar phenomena as the adsorption of large organic molecules on nanoparticle surfaces. One is the study of polymer nanocomposites,[197] [198] [199] [200] looking at changes in the conformation of polymers in a polymer melt around a nanoparticle. While this phenomenon is also about the interactions of the polymers with nanoparticles, the difference between these systems and surface adsorption is that the polymer are confined in the polymer melt as well as close to the nanoparticle surface. For the study of polymer nanocomposites, generally coarse grained methods similar to the once mentioned above are used. A second field of study which has many similarities to surface adsorption is the formation of micelles, which, depending on the size, are nanostructures as well. However instead of being confined by a surface, the systems are confined by hydrophilic/hydrophobic forces acting on different parts of the molecules. As there are only the organic molecules to simulate and not both the organic molecules and a nanoparticle, many all-atom (or united atom, treating all but the hydrogen atoms explicitly) simulations of micelles can be found,[201] [202] although coarse grained simulations on larger micelle structures exist as well.[203] [204]

\subsubsection{Interactions of Nanoscale Structures}

In most applications, nanostructures and particles have a tendency to interact, agglomerate and even coalesce or sinter, thus changing their properties and behavior. This process has been studied by several authors for the case of gaseous phase synthesis where the influence of the surrounding phase on the interaction between particles is negligible and the interaction between small nanoparticles can be simulated in vacuum.[130] [205] Other systems, such as nanoparticles in suspension, are more complicated. Typical biological media for instance are complex electrolytes containing a high concentration of organic molecules. Thus, the interaction between nanoparticles are not only the result of van der Waals and, depending on the material, magnetic forces, mitigated 
by the surrounding media, but also of electrostatic forces including the electrical double layer and the contribution of any organic molecules adsorbed at the nanoparticle surface. Due to lack of alternatives, the electrical double layer and the electrostatic interaction of nanoparticles in an electrolyte is often described by analytical theories developed for large particles where the surface can be considered to be flat locally.[112] [113] [206] [207] However both mean field calculations[208] [209] and coarse grained Monte Carlo simulations [210] [211] have shown that the ionic distribution around a nanoparticle can differ significantly from that of a flat surface. Nevertheless only two of the studies mentioned above also look at how the changes in the ionic distribution around the nanoparticle effect the interparticle forces[208] and there are still no readily available models that would be generally applicable to describe the electrostatic forces between nanoparticles. The situation is similar in for the effect of adsorbed organic molecules on the interparticle forces. There are some models available,[112] however they have been developed for larger particles and it is unclear how this is applicable to nanoparticles. The coarse grained studies that exist in literature about the interaction of polymer decorated nanoparticles,[156] [212] while very interesting, do not permit a general view of the contribution of adsorbed organic molecules on the interaction of nanoparticles.

\subsection{Summary and Conclusions}

In summary we can say that the interaction between materials and biological systems are mainly controlled by surface properties. Here computational methods can be used to clarify the surface properties of different materials, such as the morphology and the type of species and surface groups present in different environments. Typically biological media are strong electrolyte with large organic molecules, such as proteins, in suspension. The adsorption of proteins and other biomolecules at the surface will largely determine the biological interactions and there understanding is therefore of primordial importance. The adsorption of large molecules is determined by the interactions between the surface and different functional groups of the molecules, confinement and entanglement effects of the large molecules at the surface as well as by electrostatic forces. The computational study of the first two aspect is quite well established, using e.g. atomistic methods for the first and coarse grained for the second. However the interdependence between the electrical double layer and the surface adsorption of large biological molecules is often neglected. To take this into account there are two main methods (1) coarse grained Metropolis or Grand Canonical Monte Carlo methods, where the organic molecules are treated as polyelectrolytes, and (2) mean field theory.

These are the method of choice as, due to the complexity and size of the system to study, atomistic methods are not adapted. Instead a multiscale approach to study different aspects with different computational techniques is the way to get a complete picture. Finally nanoscale systems will often behave differently from bulk systems and thus have to be studied especially. 


\section{Conclusions}

In summary one can say that, although the field of solid-state computational chemistry of nanoscale biomaterials has a high potential and is rapidly growing, it is still a comparatively young field and a lot of issues still need to be addressed for a better fundamental understanding of these systems. Marco to sort out 


\section{References}

1. Rignanese, G.M., Dielectric properties of crystalline and amorphous transition metal oxides and silicates as potential high- $k$ candidates: the contribution of density-functional theory. Journal of Physics: Condensed Matter, 2005. 17(7): p. R357.

2. Godlewski, M., et al., High-k oxides by atomic layer deposition-Applications in biology and medicine. Journal of Vacuum Science \& Technology A, 2017. 35(2): p. 021508.

3. Memarzadeh, K., et al., Nanoparticulate zinc oxide as a coating material for orthopedic and dental implants. Journal of Biomedical Materials Research Part A, 2015. 103(3): p. 981-989.

4. Zhang, Y., et al., Biomedical Applications of Zinc Oxide Nanomaterials. Current Molecular Medicine, 2013. 13(10): p. 1633-1645.

5. Urban, B.E., et al., Bioimaging Using the Optimized Nonlinear Optical Properties of ZnO Nanoparticles. IEEE Journal of Selected Topics in Quantum Electronics, 2012. 18(4): p. 1451-1456.

6. Rai, M., A. Yadav, and A. Gade, Silver nanoparticles as a new generation of antimicrobials. Biotechnology Advances, 2009. 27(1): p. 76-83.

7. Dong, H.S. and S.J. Qi, Realising the potential of graphene-based materials for biosurfaces - A future perspective. Biosurface and Biotribology, 2015. 1(4): p. 229-248.

8. Kumar, S. and K. Chatterjee, Comprehensive Review on the Use of Graphene-Based Substrates for Regenerative Medicine and Biomedical Devices. ACS Applied Materials \& Interfaces, 2016. 8(40): p. 26431-26457.

9. Li, M., et al., An overview of graphene-based hydroxyapatite composites for orthopedic applications. Bioactive Materials, 2018. 3(1): p. 1-18.

10. Shi, Y.Y., et al., Electrophoretic deposition of graphene oxide reinforced chitosan-hydroxyapatite nanocomposite coatings on Ti substrate. Journal of Materials Science: Materials in Medicine, 2016. 27(3): p. 48.

11. Xu, M., D. Fujita, and N. Hanagata, Perspectives and Challenges of Emerging Single-Molecule DNA Sequencing Technologies. Small, 2009. 5(23): p. 2638-2649.

12. Yang, N. and X. Jiang, Nanocarbons for DNA sequencing: A review. Carbon, 2017. 115: p. 293-311.

13. Fabbro, A., et al., Graphene-Based Interfaces Do Not Alter Target Nerve Cells. ACS Nano, 2016. 10(1): p. 615-623.

14. Tayran, $C$., et al., Structural and electronic properties of $A B$ - and AA-stacking bilayer-graphene intercalated by $\mathrm{Li}, \mathrm{Na}, \mathrm{Ca}, \mathrm{B}, \mathrm{Al}, \mathrm{Si}, \mathrm{Ge}, \mathrm{Ag}$, and Au atoms. Solid State Communications, 2016. 231232: p. 57-63.

15. Santos, P.V., et al., Acousto-electric transport in epitaxial monolayer graphene on SiC. Applied Physics Letters, 2013. 102(22): p. 221907.

16. Donck, M.V.d., et al., Piezoelectricity in asymmetrically strained bilayer graphene. 2D Materials, 2016. 3(3): p. 035015.

17. Li, S.-Y., et al., Splitting of Van Hove singularities in slightly twisted bilayer graphene. Physical Review B, 2017. 96(15): p. 155416.

18. da Cunha Rodrigues, G., et al., Strong piezoelectricity in single-layer graphene deposited on SiO2 grating substrates. Nature Communications, 2015. 6: p. 7572.

19. Yan, H., et al., Superlattice Dirac points and space-dependent Fermi velocity in a corrugated graphene monolayer. Physical Review B, 2013. 87(7): p. 075405.

20. Ahmad, K., et al., Enhanced thermoelectric performance of Bi2Te3 based graphene nanocomposites. Applied Surface Science, 2018.

21. Dhall, A. and W. Self, Cerium Oxide Nanoparticles: A Brief Review of Their Synthesis Methods and Biomedical Applications. Antioxidants, 2018. 7(8): p. 97.

22. Hohenberg, P. and W. Kohn, Inhomogeneous Electron Gas. Physical Review, 1964. 136(3B): p. B864B871.

23. Chandraboss, V.L., B. Karthikeyan, and S. Senthilvelan, Experimental and first-principles study of guanine adsorption on ZnO clusters. Physical Chemistry Chemical Physics, 2014. 16(42): p. 2346123475.

24. Marques, M.A.L. and E.K.U. Gross, TIME-DEPENDENT DENSITY FUNCTIONAL THEORY. Annual Review of Physical Chemistry, 2004. 55(1): p. 427-455. 
25. Hedin, L., New Method for Calculating the One-Particle Green's Function with Application to the Electron-Gas Problem. Physical Review, 1965. 139(3A): p. A796-A823.

26. Salpeter, E.E. and H.A. Bethe, A Relativistic Equation for Bound-State Problems. Physical Review, 1951. 84(6): p. 1232-1242.

27. Rigamonti, S., et al., Estimating Excitonic Effects in the Absorption Spectra of Solids: Problems and Insight from a Guided Iteration Scheme. Physical Review Letters, 2015. 114(14): p. 146402.

28. Marini, A., et al., yambo: An ab initio tool for excited state calculations. Computer Physics Communications, 2009. 180(8): p. 1392-1403.

29. Milne, B.F., Enhancement of nonlinear optical properties in late group 15 tetrasubstituted cubanes. Dalton Transactions, 2014. 43(17): p. 6333-6338.

30. Attaccalite, C. and M. Grüning, Nonlinear optics from an ab initio approach by means of the dynamical Berry phase: Application to second- and third-harmonic generation in semiconductors. Physical Review B, 2013. 88(23): p. 235113.

31. Luppi, E., H. Hübener, and V. Véniard, Ab initio second-order nonlinear optics in solids: Secondharmonic generation spectroscopy from time-dependent density-functional theory. Physical Review B, 2010. 82(23): p. 235201.

32. Balasaheb, J.N., C. Sunil, and B. Vaishali, Study of electronic and optical properties of ZnO clusters using TDDFT method. Materials Research Express, 2017. 4(10): p. 106304.

33. Kurtz, H.A., J.J.P. Stewart, and K.M. Dieter, Calculation of the nonlinear optical properties of molecules. Journal of Computational Chemistry, 1990. 11(1): p. 82-87.

34. Peña-Bahamonde, J., et al., Recent advances in graphene-based biosensor technology with applications in life sciences. Journal of Nanobiotechnology, 2018. 16(1): p. 75.

35. Jiang, T., et al., Gate-tunable third-order nonlinear optical response of massless Dirac fermions in graphene. Nature Photonics, 2018. 12(7): p. 430-436.

36. Kuzmin, N.V., et al., Third harmonic generation imaging for fast, label-free pathology of human brain tumors. Biomedical Optics Express, 2016. 7(5): p. 1889-1904.

37. Malard, L.M., et al., Raman spectroscopy in graphene. Physics Reports, 2009. 473(5): p. 51-87.

38. Popov, V.N., Two-phonon Raman bands of bilayer graphene: Revisited. Carbon, 2015. 91: p. 436-444.

39. Jin-Wu, J., et al., A review on the flexural mode of graphene: lattice dynamics, thermal conduction, thermal expansion, elasticity and nanomechanical resonance. Journal of Physics: Condensed Matter, 2015. 27(8): p. 083001.

40. Amorim, B. and F. Guinea, Flexural mode of graphene on a substrate. Physical Review B, 2013. 88(11): p. 115418.

41. Kohn, W., Image of the Fermi Surface in the Vibration Spectrum of a Metal. Physical Review Letters, 1959. 2(9): p. 393-394.

42. Milošević, I., et al., Kohn anomaly in graphene. Materials Science and Engineering: B, 2011. 176(6): p. 510-511.

43. El-Kelany, K.E., et al., Piezoelectricity of Functionalized Graphene: A Quantum-Mechanical Rationalization. The Journal of Physical Chemistry C, 2016. 120(14): p. 7795-7803.

44. More, N. and G. Kapusetti, Piezoelectric material - A promising approach for bone and cartilage regeneration. Medical Hypotheses, 2017. 108: p. 10-16.

45. Chapelon, J.-Y., et al., New piezoelectric transducers for therapeutic ultrasound. Ultrasound in Medicine \& Biology, 2000. 26(1): p. 153-159.

46. Ferreira, G.N.M., A.-C. da-Silva, and B. Tomé, Acoustic wave biosensors: physical models and biological applications of quartz crystal microbalance. Trends in Biotechnology, 2009. 27(12): p. 689697.

47. Rodzinski, A., et al., Targeted and controlled anticancer drug delivery and release with magnetoelectric nanoparticles. Scientific Reports, 2016. 6: p. 20867.

48. Bassett, C.A.L. and R.O. Becker, Generation of Electric Potentials by Bone in Response to Mechanical Stress. Science, 1962. 137(3535): p. 1063.

49. Nuccio, L., L. Schulz, and A.J. Drew, Muon spin spectroscopy: magnetism, soft matter and the bridge between the two. Journal of Physics D: Applied Physics, 2014. 47(47): p. 473001. 
50. Nagamine, K., Muon Application to Advanced Bio- and Nano-Sciences. AIP Conference Proceedings, 2008. 981(1): p. 375-377.

51. Dehn, M.H., et al., Communication: Chemisorption of muonium on gold nanoparticles: A sensitive new probe of surface magnetism and reactivity. The Journal of Chemical Physics, 2016. 145(18): $p$. 181102.

52. Chen, H.M., J.D. van Horn, and Y.C. Jean, Applications of Positron Annihilation Spectroscopy to Life Science. Defect and Diffusion Forum, 2012. 331: p. 275-293.

53. Pawel, M., et al., Physics in Medicine and Biology, 2019.

54. Walle, C.G.V.d. and J. Neugebauer, HYDROGEN IN SEMICONDUCTORS. Annual Review of Materials Research, 2006. 36(1): p. 179-198.

55. Silva, E.L., et al., Hydrogen impurity in yttria: $A b$ initio and \$lensuremath\{|mu\}\$SR perspectives. Physical Review B, 2012. 85(16): p. 165211.

56. da Silva, E.L., et al., Electronic structure of interstitial hydrogen in lutetium oxide from $\$ \mid$ text $\{D F T\}+U \$$ calculations and comparison study with \$lensuremath\{|mu\}|mathrm\{SR\}\$ spectroscopy. Physical Review B, 2016. 94(1): p. 014104.

57. Makkonen, I., M. Hakala, and M.J. Puska, First-principles calculation of positron states and annihilation at defects in semiconductors. Physica B: Condensed Matter, 2006. 376-377: p. 971-974.

58. Riccò, M., et al., Muons Probe Strong Hydrogen Interactions with Defective Graphene. Nano Letters, 2011. 11(11): p. 4919-4922.

59. Park, S., et al., Hybrid functional study on structural and electronic properties of oxides. Current Applied Physics, 2011. 11(3, Supplement): p. S337-S340.

60. Hybertsen, M.S. and S.G. Louie, Electron correlation in semiconductors and insulators: Band gaps and quasiparticle energies. Physical Review B, 1986. 34(8): p. 5390-5413.

61. van Schilfgaarde, M., T. Kotani, and S. Faleev, Quasiparticle Self-Consistent \$GW\$ Theory. Physical Review Letters, 2006. 96(22): p. 226402.

62. Cox, S.F.J., et al., Oxide muonics: Il. Modelling the electrical activity of hydrogen in wide-gap and highpermittivity dielectrics. Journal of Physics: Condensed Matter, 2006. 18(3): p. 1079.

63. Andelman, T., et al., Synthesis and Cytotoxicity of Y2O3 Nanoparticles of Various Morphologies. Nanoscale Research Letters, 2009. 5(2): p. 263.

64. den Engelsen, D., et al., Ultraviolet and blue cathodoluminescence from cubic Y2O3 and Y2O3:Eu3+ generated in a transmission electron microscope. Journal of Materials Chemistry C, 2016. 4(29): $p$. 7026-7034.

65. Xu, W., Y. Chang, and G.H. Lee, Biomedical Applications of Lanthanide Oxide Nanoparticles. Journal of Biomaterials and Tissue Engineering, 2017. 7(9): p. 757-769.

66. Spasojević, I., Electron Paramagnetic Resonance - A Powerful Tool of Medical Biochemistry in Discovering Mechanisms of Disease and Treatment Prospects. 2010. 29(3): p. 175.

67. Van de Walle, C.G., Hydrogen as a Cause of Doping in Zinc Oxide. Physical Review Letters, 2000. 85(5): p. 1012-1015.

68. Li, H. and J. Robertson, Behaviour of hydrogen in wide band gap oxides. Journal of Applied Physics, 2014. 115(20): p. 203708.

69. Szász, K., et al., Hyperfine coupling of point defects in semiconductors by hybrid density functional calculations: The role of core spin polarization. Physical Review B, 2013. 88(7): p. 075202.

70. Risto, M.N., Issues in first-principles calculations for defects in semiconductors and oxides. Modelling and Simulation in Materials Science and Engineering, 2009. 17(8): p. 084001.

71. JÓNSSON, H., G. MILLS, and K.W. JACOBSEN, Nudged elastic band method for finding minimum energy paths of transitions, in Classical and Quantum Dynamics in Condensed Phase Simulations. $p$. 385-404.

72. Marinopoulos, A.G., Protons in cubic yttria-stabilized zirconia: Binding sites and migration pathways. Solid State lonics, 2018. 315: p. 116-125.

73. Nanda, B.R.K., et al., Electronic structure of the substitutional vacancy in graphene: density-functional and Green's function studies. New Journal of Physics, 2012. 14(8): p. 083004.

74. Akmalova, Y.A., et al., Positron Lifetime Spectroscopy of Silicon Nanocontainers for Cancer Theranostic Applications. 2018, 2018: p. 9. 
75. Puska, M.J. and R.M. Nieminen, Theory of positrons in solids and on solid surfaces. Reviews of Modern Physics, 1994. 66(3): p. 841-897.

76. Chirayath, V.A., et al., Investigation of graphene using low energy positron annihilation induced Doppler broadening spectroscopy. Journal of Physics: Conference Series, 2017. 791(1): p. 012032.

77. Billemont, P., B. Coasne, and G. De Weireld, Adsorption of Carbon Dioxide, Methane, and Their Mixtures in Porous Carbons: Effect of Surface Chemistry, Water Content, and Pore Disorder. Langmuir, 2013. 29(10): p. 3328-3338.

78. Allen, J.P., et al., Atomistic modelling of adsorption and segregation at inorganic solid interfaces. Molecular Simulation, 2009. 35(7): p. 584-608.

79. Aschauer, U., et al., Growth modification of hematite by phosphonate additives. Journal of Crystal Growth, 2008. 310(3): p. 688-698.

80. Galmarini, S. and P. Bowen, Atomistic simulation of the adsorption of calcium and hydroxyl ions onto portlandite surfaces - towards crystal growth mechanisms. Cement and Concrete Research, 2016. 81: p. 16-23.

81. Galmarini, S., A. Kunhi Mohamed, and P. Bowen, Atomistic Simulations of Silicate Species Interaction with Portlandite Surfaces. The Journal of Physical Chemistry C, 2016. 120(39): p. 22407-22413.

82. Tilocca, A. and A. Selloni, Reaction pathway and free energy barrier for defect-induced water dissociation on the (101) surface of TiO2-anatase. The Journal of Chemical Physics, 2003. 119(14): p. 7445-7450.

83. Aschauer, U., et al., Influence of Subsurface Defects on the Surface Reactivity of TiO2: Water on Anatase (101). The Journal of Physical Chemistry C, 2010. 114(2): p. 1278-1284.

84. Wulff, G., Zur Frage der Geschwindigkeit des Wachstums und der auflösung der Krystallflächen. Zeitschrift fur Krystallographie und Mineralogie, 1901. 34: p. 449-530.

85. Marmier, A. and S.C. Parker, $A b$ initio morphology and surface thermodynamics of \$lensuremath\{|alpha\}|ensuremath\{-\}\{|mathrm\{A|\}\}_\{2\}\{|mathrm\{O\}\}_\{3\}\$. Physical Review B, 2004. 69(11): p. 115409.

86. Arrouvel, C., et al., Effects of morphology on surface hydroxyl concentration: a DFT comparison of anatase-TiO2 and y-alumina catalytic supports. Journal of Catalysis, 2004. 222(1): p. 152-166.

87. Vitos, L., et al., The surface energy of metals. Surface Science, 1998. 411(1): p. 186-202.

88. Tasker, P.W., SURFACE ENERGIES, SURFACE TENSIONS AND SURFACE-STRUCTURE OF THE ALKALIHALIDE CRYSTALS. Philosophical Magazine A: Physics of Condensed Matter Structure Defects and Mechanical Properties, 1979. 39(2): p. 119-136.

89. Aschauer, U., P. Bowen, and S.C. Parker, Atomistic Modeling Study of Surface Segregation in Nd:YAG. Journal of the American Ceramic Society, 2006. 89(12): p. 3812-3816.

90. Galmarini, S., et al., Changes in portlandite morphology with solvent composition: Atomistic simulations and experiment. Cement and Concrete Research, 2011. 41(12): p. 1330-1338.

91. de Leeuw, N.H. and S.C. Parker, Surface Structure and Morphology of Calcium Carbonate Polymorphs Calcite, Aragonite, and Vaterite: An Atomistic Approach. The Journal of Physical Chemistry B, 1998. 102(16): p. 2914-2922.

92. Schardt, J., et al., Crystallography of the $\$(3 \mid$ ifmmodeltimes|elseltexttimes|fi\{\}3)\$ surface reconstruction of $\$ 3 C$ lensuremath $\{-\} \mid$ mathrm $\{\mathrm{SiC}\}(111), \$ \$ 4 \mathrm{H}$ |ensuremath $\{-\} \mid$ mathrm $\{\mathrm{SiC}\}(0001), \$$ and $\$ 6 \mathrm{H} \mid$ ensuremath $\{-\} \mid$ mathrm $\{\mathrm{SiC}\}(0001) \$$ surfaces retrieved by low-energy electron diffraction. Physical Review B, 2000. 62(15): p. 10335-10344.

93. Broughton, J.Q. and G.H. Gilmer, Molecular dynamics investigation of the crystal-fluid interface. VI. Excess surface free energies of crystal-liquid systems. The Journal of Chemical Physics, 1986. 84(10): p. 5759-5768.

94. Davidchack, R.L. and B.B. Laird, Crystal Structure and Interaction Dependence of the Crystal-Melt Interfacial Free Energy. Physical Review Letters, 2005. 94(8): p. 086102.

95. Grochola, G., et al., "Exact" surface free energies of iron surfaces using a modified embedded atom method potential and $\lambda$ integration. The Journal of Chemical Physics, 2004. 120(7): p. 3425-3430.

96. Asta, M., J.J. Hoyt, and A. Karma, Calculation of alloy solid-liquid interfacial free energies from atomicscale simulations. Physical Review B, 2002. 66(10): p. 100101. 
97. Becker, C.A., et al., Atomistic Underpinnings for Orientation Selection in Alloy Dendritic Growth. Physical Review Letters, 2007. 98(12): p. 125701.

98. Davidchack, R.L., J.R. Morris, and B.B. Laird, The anisotropic hard-sphere crystal-melt interfacial free energy from fluctuations. The Journal of Chemical Physics, 2006. 125(9): p. 094710.

99. Morris, J.R., M.I. Mendelev, and D.J. Srolovitz, A comparison of crystal-melt interfacial free energies using different Al potentials. Journal of Non-Crystalline Solids, 2007. 353(32): p. 3565-3569.

100. Asta, M., et al., Solidification microstructures and solid-state parallels: Recent developments, future directions. Acta Materialia, 2009. 57(4): p. 941-971.

101. Kerisit, S., et al., Molecular dynamics simulations of the interactions between water and inorganic solids. Journal of Materials Chemistry, 2005. 15(14): p. 1454-1462.

102. Claus, M., et al., Water adsorption on amorphous silica surfaces: a Car-Parrinello simulation study. Journal of Physics: Condensed Matter, 2005. 17(26): p. 4005.

103. Foucaud, Y., et al., Surface Properties of Fluorite in Presence of Water: An Atomistic Investigation. The Journal of Physical Chemistry B, 2018. 122(26): p. 6829-6836.

104. Churakov, S.V., et al., Intrinsic Acidity of Surface Sites in Calcium Silicate Hydrates and Its Implication to Their Electrokinetic Properties. The Journal of Physical Chemistry C, 2014. 118(22): p. 11752-11762.

105. Aykol, M. and K.A. Persson, Oxidation Protection with Amorphous Surface Oxides: Thermodynamic Insights from Ab Initio Simulations on Aluminum. ACS Applied Materials \& Interfaces, 2018. 10(3): p. 3039-3045.

106. Galmarini, S., et al., Atomistic Simulation of $Y$-Doped alpha-Alumina Interfaces. Journal of the American Ceramic Society, 2008. 91(11): p. 3643-3651.

107. Kerisit, S., et al., Atomistic simulation of charged iron oxyhydroxide surfaces in contact with aqueous solution. Chemical Communications, 2005(24): p. 3027-3029.

108. Mackrodt, W.C. and P.W. Tasker, Segregation Isotherms at the Surfaces of Oxides. Journal of the American Ceramic Society, 1989. 72(9): p. 1576-1583.

109. Purton, J.A., J.C. Crabtree, and S.C. Parker, DL_MONTE: a general purpose program for parallel Monte Carlo simulation. Molecular Simulation, 2013. 39(14-15): p. 1240-1252.

110. Desu, S.B. and D.A. Payne, Interfacial Segregation in Perovskites: I, Theory. Journal of the American Ceramic Society, 1990. 73(11): p. 3391-3397.

111. Yan, M.F., R.M. Cannon, and H.K. Bowen, Space charge, elastic field, and dipole contributions to equilibrium solute segregation at interfaces. Journal of Applied Physics, 1983. 54(2): p. 764-778.

112. Burgos-Montes, O., R. Moreno, and P. Bowen, Hamaker 2: A Toolkit for the Calculation of Particle Interactions and Suspension Stability and its Application to Mullite Synthesis by Colloidal Methods AU - Aschauer, Ulrich. Journal of Dispersion Science and Technology, 2011. 32(4): p. 470-479.

113. Behrens, S.H. and M. Borkovec, Electrostatic Interaction of Colloidal Surfaces with Variable Charge. The Journal of Physical Chemistry B, 1999. 103(15): p. 2918-2928.

114. Forsman, J., et al., Attractive Surface Forces Due to Liquid Density Depression. The Journal of Physical Chemistry B, 1997. 101(21): p. 4253-4259.

115. Labbez, C., et al., C-S-H/solution interface: Experimental and Monte Carlo studies. Cement and Concrete Research, 2011. 41(2): p. 161-168.

116. Freschauf, L.R., et al., Shrink-Induced Superhydrophobic and Antibacterial Surfaces in Consumer Plastics. PLOS ONE, 2012. 7(8): p. e40987.

117. Wang, H., et al., High antibacterial efficiency of pDMAEMA modified silicon nanowire arrays. Colloids and Surfaces B: Biointerfaces, 2011. 83(2): p. 355-359.

118. Zarzycki, P., S. Kerisit, and K.M. Rosso, Molecular Dynamics Study of the Electrical Double Layer at Silver Chloride-Electrolyte Interfaces. The Journal of Physical Chemistry C, 2010. 114(19): p. 89058916.

119. Fan, H.J., et al., Influence of Surface Diffusion on the Formation of Hollow Nanostructures Induced by the Kirkendall Effect: The Basic Concept. Nano Letters, 2007. 7(4): p. 993-997.

120. Smirnov, K.S. and D. Bougeard, A Molecular Dynamics Study of Structure and Short-time Dynamics of Water in Kaolinite. The Journal of Physical Chemistry B, 1999. 103(25): p. 5266-5273.

121. Aschauer, U., P. Bowen, and S.C. Parker, Oxygen vacancy diffusion in alumina: New atomistic simulation methods applied to an old problem. Acta Materialia, 2009. 57(16): p. 4765-4772. 
122. Tewari, A., U. Aschauer, and P. Bowen, Atomistic Modeling of Effect of $\mathrm{Mg}$ on Oxygen Vacancy Diffusion in $\alpha$-Alumina. Journal of the American Ceramic Society, 2014. 97(8): p. 2596-2601.

123. Frank, F.C., The influence of dislocations on crystal growth. Discussions of the Faraday Society, 1949. 5(0): p. 48-54.

124. Chen, L., et al., Screw-Dislocation-Driven Growth of Two-Dimensional Few-Layer and Pyramid-like WSe2 by Sulfur-Assisted Chemical Vapor Deposition. ACS Nano, 2014. 8(11): p. 11543-11551.

125. Chu, Y., et al., Engineer in Situ Growth of $\alpha$-Al2O3 Whiskers by Axial Screw Dislocations. Crystal Growth \& Design, 2017. 17(4): p. 1999-2005.

126. Ding, F., A.R. Harutyunyan, and B.I. Yakobson, Dislocation theory of chirality-controlled nanotube growth. Proceedings of the National Academy of Sciences, 2009. 106(8): p. 2506.

127. Piana, S., M. Reyhani, and J.D. Gale, Simulating micrometre-scale crystal growth from solution. Nature, 2005. 438: p. 70.

128. Cölfen, H. and S. Mann, Higher-Order Organization by Mesoscale Self-Assembly and Transformation of Hybrid Nanostructures. Angewandte Chemie International Edition, 2003. 42(21): p. 2350-2365.

129. Penn, R.L. and J.F. Banfield, Imperfect Oriented Attachment: Dislocation Generation in Defect-Free Nanocrystals. Science, 1998. 281(5379): p. 969.

130. Spagnoli, D., J.F. Banfield, and S.C. Parker, Free energy change of aggregation of nanoparticles. Journal of Physical Chemistry C, 2008. 112(38): p. 14731-14736.

131. von Moos, N., et al., Pro-oxidant effects of nano-TiO2 on Chlamydomonas reinhardtii during shortterm exposure. RSC Advances, 2016. 6(116): p. 115271-115283.

132. Ramamoorthy, M., D. Vanderbilt, and R.D. King-Smith, First-principles calculations of the energetics of stoichiometric $\{\{\mid$ mathrm\{TiO\}\}_\{2\}\$ surfaces. Physical Review B, 1994. 49(23): p. 16721-16727.

133. Perron, H., et al., Optimisation of accurate rutile TiO2 (110), (100), (101) and (001) surface models from periodic DFT calculations. Theoretical Chemistry Accounts, 2007. 117(4): p. 565-574.

134. Elliott, S.D. and S.P. Bates, Assignment of the $\$(1 \mid$ ifmmodeltimeslelse|texttimes (fi \{\} 2$) \$$ surface of rutile $\{\{\mid$ mathrm $\{$ TiO $\}\}\{2\}(110) \$$ from first principles. Physical Review B, 2003. 67(3): p. 035421.

135. Cheng, H. and A. Selloni, Surface and subsurface oxygen vacancies in anatase $\$\{\mid$ text $\{$ TiO $\}\}\{2\} \$$ and differences with rutile. Physical Review B, 2009. 79(9): p. 092101.

136. Coad, B.R., et al., Biomaterials surfaces capable of resisting fungal attachment and biofilm formation. Biotechnology Advances, 2014. 32(2): p. 296-307.

137. Alexis, F., et al., Factors Affecting the Clearance and Biodistribution of Polymeric Nanoparticles. Molecular Pharmaceutics, 2008. 5(4): p. 505-515.

138. Maurizi, L., et al., Influence of Surface Charge and Polymer Coating on Internalization and Biodistribution of Polyethylene Glycol-Modified Iron Oxide Nanoparticles. Journal of Biomedical Nanotechnology, 2015. 11(1): p. 126-136.

139. Yamaura, M., et al., Preparation and characterization of (3-aminopropyl)triethoxysilane-coated magnetite nanoparticles. Journal of Magnetism and Magnetic Materials, 2004. 279(2): p. 210-217.

140. Dobrovolskaia, M.A., et al., Interaction of colloidal gold nanoparticles with human blood: effects on particle size and analysis of plasma protein binding profiles. Nanomedicine: Nanotechnology, Biology and Medicine, 2009. 5(2): p. 106-117.

141. Vroman, L., Effect of Adsorbed Proteins on the Wettability of Hydrophilic and Hydrophobic Solids. Nature, 1962. 196: p. 476.

142. Bertrand, N., et al., Mechanistic understanding of in vivo protein corona formation on polymeric nanoparticles and impact on pharmacokinetics. Nature Communications, 2017. 8(1): p. 777.

143. Galmarini, S., et al., Beyond Unpredictability: The Importance of Reproducibility in Understanding the Protein Corona of Nanoparticles. Bioconjugate Chemistry, 2018. 29(10): p. 3385-3393.

144. Sakulkhu, U., et al., Ex situ evaluation of the composition of protein corona of intravenously injected superparamagnetic nanoparticles in rats. Nanoscale, 2014. 6(19): p. 11439-11450.

145. Aschauer, U., et al., Growth Modification of Seeded Calcite by Carboxylic Acid Oligomers and Polymers: Toward an Understanding of Complex Growth Mechanisms. Crystal Growth \& Design, 2010. 10(9): p. 3956-3963. 
146. Penna, M.J., M. Mijajlovic, and M.J. Biggs, Molecular-Level Understanding of Protein Adsorption at the Interface between Water and a Strongly Interacting Uncharged Solid Surface. Journal of the American Chemical Society, 2014. 136(14): p. 5323-5331.

147. Utesch, T., G. Daminelli, and M.A. Mroginski, Molecular Dynamics Simulations of the Adsorption of Bone Morphogenetic Protein-2 on Surfaces with Medical Relevance. Langmuir, 2011. 27(21): p. 13144-13153.

148. Bonvin, D., et al., Versatility of Pyridoxal Phosphate as a Coating of Iron Oxide Nanoparticles. Nanomaterials, 2017. 7(8): p. 202.

149. Bonvin, D., et al., Protein Corona: Impact of Lymph Versus Blood in a Complex In Vitro Environment. Small, 2017. 13(29): p. 1700409.

150. Bertran, O., et al., Modeling Nanosized Single Molecule Objects: Dendronized Polymers Adsorbed onto Mica. The Journal of Physical Chemistry C, 2015. 119(7): p. 3746-3753.

151. Wei, T., M.A. Carignano, and I. Szleifer, Lysozyme Adsorption on Polyethylene Surfaces: Why Are Long Simulations Needed? Langmuir, 2011. 27(19): p. 12074-12081.

152. Hassan, C.M., P. Trakampan, and N.A. Peppas, Water Solubility Characteristics of Poly(vinyl alcohol) and Gels Prepared by Freezing/Thawing Processes, in Water Soluble Polymers: Solutions Properties and Applications, Z. Amjad, Editor 2002, Springer US: Boston, MA. p. 31-40.

153. Wei, S., L.S. Ahlstrom, and C.L. Brooks III, Exploring Protein-Nanoparticle Interactions with CoarseGrained Protein Folding Models. Small, 2017. 13(18): p. 1603748.

154. Doyle, P.S., E.S.G. Shaqfeh, and A.P. Gast, Rheology of Polymer Brushes: A Brownian Dynamics Study. Macromolecules, 1998. 31(16): p. 5474-5486.

155. Qi, S. and F. Schmid, Dynamic Density Functional Theories for Inhomogeneous Polymer Systems Compared to Brownian Dynamics Simulations. Macromolecules, 2017. 50(24): p. 9831-9845.

156. Modica, K.J., T.B. Martin, and A. Jayaraman, Effect of Polymer Architecture on the Structure and Interactions of Polymer Grafted Particles: Theory and Simulations. Macromolecules, 2017. 50(12): p. 4854-4866.

157. Singh, M.K., et al., Effect of Crosslinking on the Microtribological Behavior of Model Polymer Brushes. Tribology Letters, 2016. 63(2): p. 17.

158. Sheng, J. and K. Luo, Conformation and adsorption transition on an attractive surface of a ring polymer in solution. RSC Advances, 2015. 5(3): p. 2056-2061.

159. Janke, W. and W. Paul, Thermodynamics and structure of macromolecules from flat-histogram Monte Carlo simulations. Soft Matter, 2016. 12(3): p. 642-657.

160. Förster, S., et al., Polymer adsorption on reconstructed Au(001): A statistical description of P3HT by scanning tunneling microscopy and coarse-grained Monte Carlo simulations. The Journal of Chemical Physics, 2014. 141(16): p. 164701.

161. Halperin, A., M. Tirrell, and T.P. Lodge, Tethered chains in polymer microstructures, in Macromolecules: Synthesis, Order and Advanced Properties1992, Springer Berlin Heidelberg: Berlin, Heidelberg. p. 31-71.

162. Milner, S.T., Polymer Brushes. Science, 1991. 251(4996): p. 905.

163. Daoud, M. and J.P. Cotton, Star shaped polymers : a model for the conformation and its concentration dependence. J. Phys. France, 1982. 43(3): p. 531-538.

164. Kröger, M., O. Peleg, and A. Halperin, From Dendrimers to Dendronized Polymers and Forests: Scaling Theory and its Limitations. Macromolecules, 2010. 43(14): p. 6213-6224.

165. Halperin, A. and M. Kröger, Theoretical considerations on mechanisms of harvesting cells cultured on thermoresponsive polymer brushes. Biomaterials, 2012. 33(20): p. 4975-4987.

166. Halperin, A. and M. Kröger, Thermoresponsive Cell Culture Substrates Based on PNIPAM Brushes Functionalized with Adhesion Peptides: Theoretical Considerations of Mechanism and Design. Langmuir, 2012. 28(48): p. 16623-16637.

167. McNamara, J., C.Y. Kong, and M. Muthukumar, Monte Carlo studies of adsorption of a sequenced polyelectrolyte to patterned surfaces. The Journal of Chemical Physics, 2002. 117(11): p. 5354-5360.

168. Luque-Caballero, G., A. Martín-Molina, and M. Quesada-Pérez, Polyelectrolyte adsorption onto likecharged surfaces mediated by trivalent counterions: A Monte Carlo simulation study. The Journal of Chemical Physics, 2014. 140(17): p. 174701. 
169. Chen, S., et al., Surface hydration: Principles and applications toward low-fouling/nonfouling biomaterials. Polymer, 2010. 51(23): p. 5283-5293.

170. Szleifer, I., Protein Adsorption on Surfaces with Grafted Polymers: A Theoretical Approach. Biophysical Journal, 1997. 72(2, Part 1): p. 595-612.

171. Fang, F. and I. Szleifer, Controlled release of proteins from polymer-modified surfaces. Proceedings of the National Academy of Sciences, 2006. 103(15): p. 5769.

172. Nagumo, R., et al., Assessment of the Antifouling Properties of Polyzwitterions from Free Energy Calculations by Molecular Dynamics Simulations. Industrial \& Engineering Chemistry Research, 2012. 51(11): p. 4458-4462.

173. Zolk, M., et al., Solvation of Oligo(ethylene glycol)-Terminated Self-Assembled Monolayers Studied by Vibrational Sum Frequency Spectroscopy. Langmuir, 2000. 16(14): p. 5849-5852.

174. Wang, R.L.C., H.J. Kreuzer, and M. Grunze, Molecular Conformation and Solvation of Oligolethylene glycol)-Terminated Self-Assembled Monolayers and Their Resistance to Protein Adsorption. The Journal of Physical Chemistry B, 1997. 101(47): p. 9767-9773.

175. Herrwerth, S., et al., Factors that Determine the Protein Resistance of Oligoether Self-Assembled Monolayers - Internal Hydrophilicity, Terminal Hydrophilicity, and Lateral Packing Density. Journal of the American Chemical Society, 2003. 125(31): p. 9359-9366.

176. Nagasawa, D., et al., Role of Interfacial Water in Protein Adsorption onto Polymer Brushes as Studied by SFG Spectroscopy and QCM. The Journal of Physical Chemistry C, 2015. 119(30): p. 17193-17201.

177. Pertsin, A.J. and M. Grunze, Computer Simulation of Water near the Surface of Oligo(ethylene glycol)Terminated Alkanethiol Self-Assembled Monolayers. Langmuir, 2000. 16(23): p. 8829-8841.

178. Zheng, J., et al., Molecular simulation studies of the structure of phosphorylcholine self-assembled monolayers. The Journal of Chemical Physics, 2006. 125(17): p. 174714.

179. Gupta, A.K. and M. Gupta, Synthesis and surface engineering of iron oxide nanoparticles for biomedical applications. Biomaterials, 2005. 26(18): p. 3995-4021.

180. Stark, W.J., et al., Industrial applications of nanoparticles. Chemical Society Reviews, 2015. 44(16): p. 5793-5805.

181. Scotter, M., et al., Applications and implications of nanotechnologies for the food sector AU Chaudhry, Qasim. Food Additives \& Contaminants: Part A, 2008. 25(3): p. 241-258.

182. Lim, D.-H. and J. Wilcox, DFT-Based Study on Oxygen Adsorption on Defective Graphene-Supported Pt Nanoparticles. The Journal of Physical Chemistry C, 2011. 115(46): p. 22742-22747.

183. Lazzari, R., et al., Surface and Epitaxial Stresses on Supported Metal Clusters. Nano Letters, 2016. 16(4): p. 2574-2579.

184. De Angelis, F., A. Tilocca, and A. Selloni, Time-Dependent DFT Study of [Fe(CN)6]4- Sensitization of TiO2 Nanoparticles. Journal of the American Chemical Society, 2004. 126(46): p. 15024-15025.

185. Naicker, P.K., et al., Characterization of Titanium Dioxide Nanoparticles Using Molecular Dynamics Simulations. The Journal of Physical Chemistry B, 2005. 109(32): p. 15243-15249.

186. Bembel, A.G., On the Size Dependences of the Metallic Nanoparticle Evaporation and Sublimation Heats: Thermodynamics and Atomistic Modeling. Russian Physics Journal, 2017. 59(10): p. 15671574.

187. Grochola, G., S.P. Russo, and I.K. Snook, On morphologies of gold nanoparticles grown from molecular dynamics simulation. The Journal of Chemical Physics, 2007. 126(16): p. 164707.

188. Li, Y., M. Kröger, and W.K. Liu, Endocytosis of PEGylated nanoparticles accompanied by structural and free energy changes of the grafted polyethylene glycol. Biomaterials, 2014. 35(30): p. 8467-8478.

189. Wei, T.-Y., S.-Y. Lu, and Y.-C. Chang, Transparent, Hydrophobic Composite Aerogels with High Mechanical Strength and Low High-Temperature Thermal Conductivities. The Journal of Physical Chemistry B, 2008. 112(38): p. 11881-11886.

190. Trazkovich, A.J., M.F. Wendt, and L.M. Hall, Effect of copolymer sequence on structure and relaxation times near a nanoparticle surface. Soft Matter, 2018. 14(28): p. 5913-5921.

191. Kashyap, N. and B. Rai, Molecular mechanism of transdermal co-delivery of interferon-alpha protein with gold nanoparticle - a molecular dynamics study AU - Gupta, Rakesh. Molecular Simulation, 2018. 44(4): p. 274-284. 
192. Lin, J., et al., Penetration of Lipid Membranes by Gold Nanoparticles: Insights into Cellular Uptake, Cytotoxicity, and Their Relationship. ACS Nano, 2010. 4(9): p. 5421-5429.

193. Rossner, C., et al., Phase separation in mixed polymer brushes on nanoparticle surfaces enables the generation of anisotropic nanoarchitectures. Soft Matter, 2018. 14(22): p. 4551-4557.

194. Mandal, T., et al., Effect of Surface Charge and Hydrophobicity on Phospholipid-Nanoparticle Corona Formation: A Molecular Dynamics Simulation Study. Colloid and Interface Science Communications, 2018. 25: p. 7-11.

195. Chodanowski, P. and S. Stoll, Polyelectrolyte adsorption on charged particles: lonic concentration and particle size effects $-A$ Monte Carlo approach. The Journal of Chemical Physics, 2001. 115(10): p. 4951-4960.

196. Chodanowski, P. and S. Stoll, Polyelectrolyte Adsorption on Charged Particles in the Debye-Hückel Approximation. A Monte Carlo Approach. Macromolecules, 2001. 34(7): p. 2320-2328.

197. Kumar, S.K., V. Ganesan, and R.A. Riggleman, Perspective: Outstanding theoretical questions in polymer-nanoparticle hybrids. The Journal of Chemical Physics, 2017. 147(2): p. 020901.

198. Dionne, P.J., C.R. Picu, and R. Ozisik, Adsorption and Desorption Dynamics of Linear Polymer Chains to Spherical Nanoparticles: A Monte Carlo Investigation. Macromolecules, 2006. 39(8): p. 3089-3092.

199. Smith, J.S., D. Bedrov, and G.D. Smith, A molecular dynamics simulation study of nanoparticle interactions in a model polymer-nanoparticle composite. Composites Science and Technology, 2003. 63(11): p. 1599-1605.

200. Sampath, J. and L.M. Hall, Influence of a nanoparticle on the structure and dynamics of model ionomer melts. Soft Matter, 2018. 14(22): p. 4621-4632.

201. Marrink, S.J., D.P. Tieleman, and A.E. Mark, Molecular Dynamics Simulation of the Kinetics of Spontaneous Micelle Formation. The Journal of Physical Chemistry B, 2000. 104(51): p. 12165-12173.

202. Yordanova, D., et al., Solute Partitioning in Micelles: Combining Molecular Dynamics Simulations, COSMOmic, and Experiments. The Journal of Physical Chemistry B, 2017. 121(23): p. 5794-5809.

203. Bogusz, S., R.M. Venable, and R.W. Pastor, Molecular Dynamics Simulations of Octyl Glucoside Micelles: Structural Properties. The Journal of Physical Chemistry B, 2000. 104(23): p. 5462-5470.

204. Soper, A.K. and K.J. Edler, Coarse-grained empirical potential structure refinement: Application to a reverse aqueous micelle. Biochimica et Biophysica Acta (BBA) - General Subjects, 2017. 1861(6): p. 1652-1660.

205. Zachariah, M.R. and M.J. Carrier, Molecular dynamics computation of gas-phase nanoparticle sintering: a comparison with phenomenological models. Journal of Aerosol Science, 1999. 30(9): p. 1139-1151.

206. Derjaguin, B.V., A theory of the heterocoagulation, interaction and adhesion of dissimilar particles in solutions of electrolytes. Discussions of the Faraday Society, 1954. 18(0): p. 85-98.

207. Forsman, J., A Simple Correlation-Corrected Poisson-Boltzmann Theory. The Journal of Physical Chemistry B, 2004. 108(26): p. 9236-9245.

208. Ohshima, H., T.W. Healy, and L.R. White, Accurate analytic expressions for the surface charge density/surface potential relationship and double-layer potential distribution for a spherical colloidal particle. Journal of Colloid and Interface Science, 1982. 90(1): p. 17-26.

209. Pfeiffer, C., et al., Interaction of colloidal nanoparticles with their local environment: the (ionic) nanoenvironment around nanoparticles is different from bulk and determines the physico-chemical properties of the nanoparticles. Journal of The Royal Society Interface, 2014. 11(96): p. 20130931.

210. López-García, J.J., J. Horno, and C. Grosse, Ion size effects on the dielectric and electrokinetic properties in aqueous colloidal suspensions. Current Opinion in Colloid \& Interface Science, 2016. 24: p. 23-31.

211. Svensson, B. and B. Jönsson, The interaction between charged aggregates in electrolyte solution. A Monte Carlo simulation study. Chemical Physics Letters, 1984. 108(6): p. 580-584.

212. Pryamitsyn, V. and V. Ganesan, Pair interactions in polyelectrolyte-nanoparticle systems: Influence of dielectric inhomogeneities and the partial dissociation of polymers and nanoparticles. The Journal of Chemical Physics, 2015. 143(16): p. 164904. 